\title{
Raphé Neurons Stimulate Respiratory Circuit Activity by Multiple Mechanisms via Endogenously Released Serotonin and Substance P
}

\author{
Krzysztof Ptak, ${ }^{1 *}$ Tadashi Yamanishi, ${ }^{1 *}$ Jason Aungst, ${ }^{1 *}$ Lorin S. Milescu, ${ }^{1}$ Ruli Zhang, ${ }^{1}$ George B. Richerson, ${ }^{2,3,4}$ and \\ Jeffrey C. Smith ${ }^{1}$ \\ ${ }^{1}$ Cellular and Systems Neurobiology Section, Porter Neuroscience Research Center, National Institute of Neurological Disorders and Stroke-National \\ Institutes of Health, Bethesda, Maryland 20892, Departments of ${ }^{2}$ Neurology and ${ }^{3}$ Cellular and Molecular Physiology, Yale University School of Medicine, \\ New Haven, Connecticut 06520, and ${ }^{4}$ Department of Neurology, Veterans Affairs Medical Center, West Haven, Connecticut 06516
}

Brainstem serotonin (5-HT) neurons modulate activity of many neural circuits in the mammalian brain, but in many cases endogenous mechanisms have not been resolved. Here, we analyzed actions of raphé 5-HT neurons on respiratory network activity including at the level of the pre-Bötzinger complex (pre-BötC) in neonatal rat medullary slices in vitro, and in the more intact nervous system of juvenile rats in arterially perfused brainstem-spinal cord preparations in situ. At basal levels of activity, excitation of the respiratory network via simultaneous release of 5-HT and substance $\mathrm{P}(\mathrm{SP})$, acting at 5- $\mathrm{HT}_{2 \mathrm{~A} / 2 \mathrm{C}}, 5-\mathrm{HT}_{4}$, and/or neurokinin-1 receptors, was required to maintain inspiratory motor output in both the neonatal and juvenile systems. The midline raphé obscurus contained spontaneously active 5-HT neurons, some of which projected to the pre-BötC and hypoglossal motoneurons, colocalized 5-HT and SP, and received reciprocal excitatory connections from the pre-BötC. Experimentally augmenting raphé obscurus activity increased motor output by simultaneously exciting pre-BötC and motor neurons. Biophysical analyses in vitro demonstrated that 5-HT and SP modulated background cation conductances in pre-BötC and motor neurons, including a nonselective cation leak current that contributed to the resting potential, which explains the neuronal depolarization that augmented motor output. Furthermore, we found that 5-HT, but not SP, can transform the electrophysiological phenotype of some pre-BötC neurons to intrinsic bursters, providing 5-HT with an additional role in promoting rhythm generation. We conclude that raphé 5-HT neurons excite key circuit components required for generation of respiratory motor output.

\section{Introduction}

Serotonin (5-HT) neurons regulate many brain functions (Jacobs and Azmitia, 1992), including autonomic and motor function, such as the neural control of breathing. In the control of breathing, these neurons have been proposed to play diverse roles, including in central $\mathrm{CO}_{2} / \mathrm{pH}$ chemoreception (Richerson, 2004), sleep-wake state control, and adaptive changes in respiratory motor output (Feldman et al., 2003). Furthermore, the raphé system has been implicated in the pathophysiology of sleep apnea (Kubin et al., 1998), sudden infant death syndrome (SIDS) (Paterson et al., 2006), and other disorders associated with unstable breathing (Richter et al., 2003).

The modulatory actions of specific subpopulations of 5-HT neurons on critical respiratory circuit components controlling

\footnotetext{
Received Nov. 1, 2008; revised Dec. 15, 2008; accepted Feb. 13, 2009.

This research was supported by the Intramural Research Program of the National Institutes of Health-National Institute of Neurological Disorders and Stroke and in part by Grant P01HD36379 from the National Institutes of Health, and by grants from the Veterans Affairs Medical Center and Bumpus Foundation.

*K.P., T.Y., and J.A. contributed equally to this work.

Correspondence should be addressed to Dr. Jeffrey C. Smith, Porter Neuroscience Research Center, Building 35, Room 3C-917, 35 Convent Drive, National Institute of Neurological Disorders and Stroke-National Institutes of Health, Bethesda, MD 20892. E-mail: jsmith@helix.nih.gov.

D01:10.1523/JNEUROSCI.5271-08.2009

Copyright $\odot 2009$ Society for Neuroscience $\quad$ 0270-6474/09/293720-18\$15.00/0
}

breathing have not been clearly established. Indeed, it has been debated whether the primary effect of raphé neurons on breathing is inhibitory or excitatory (Richter et al., 2003; Richerson, 2004). There has also been controversy regarding the roles of various 5-HT receptor subtypes at different stages of development (Toppin et al., 2007). Finally, while NK-1 receptors are known to be important for respiratory rhythm generation (Feldman et al., 2003), the endogenous source of substance P (SP) has not been established, but raphé neurons that colocalize SP are a likely source.

We resolved some of these issues by analyzing the role of the raphé system using in vitro neonatal rat medullary slices containing the pre-Bötzinger complex (pre-BötC) (Smith et al., 1991; Koizumi et al., 2008) and the more intact arterially perfused in situ brainstem-spinal cords of juvenile rats (Paton, 1996; Smith et al., 2007). We investigated the role of activity of raphé neurons and endogenous transmitter release, particularly contributions of a specific raphé subpopulation, the raphé obscurus, which has been implicated in control of breathing, including involvement in pathophysiological conditions (Feldman et al., 2003; Paterson et al., 2006). Our experiments revealed a number of novel results, including the following: (1) all medullary slices that generate respiratory motor output contain many spontaneously active $5-\mathrm{HT}$ 

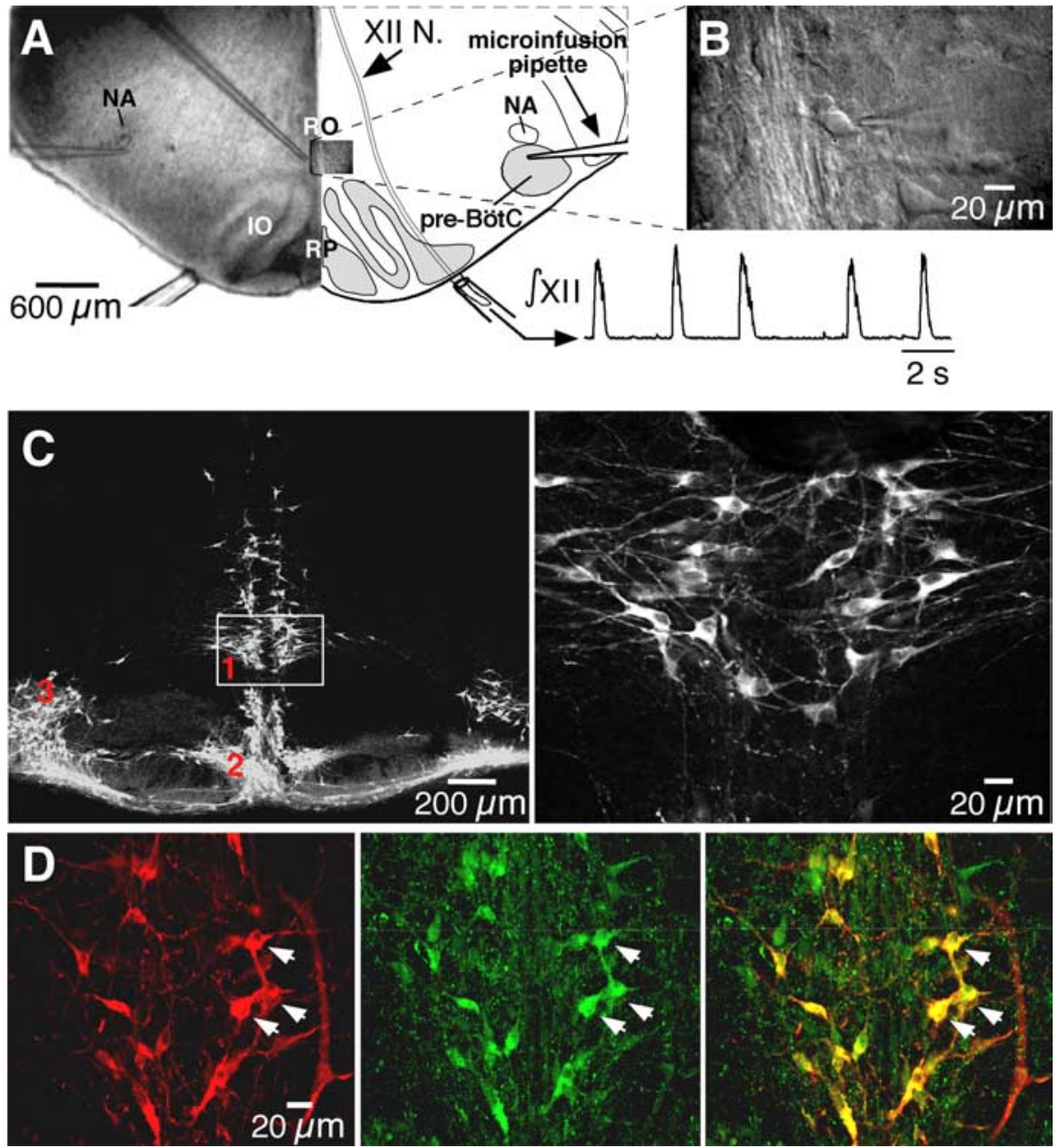

Figure 1. Rhythmogenic medullary slices contain neurons immunoreactive for 5-HT and substance P. A, Low-power $(5 \times)$ IR-DIC image (left) and schematic (right) of medullary slice preparation showing configuration with microinfusion pipettes in the n. raphé obscurus (RO) or bilaterally in the pre-BötC. Inspiratory activity was recorded from XII nerve (integrated signal, $\int$ XII) with suction electrodes. I0, Inferior olive; NA, semicompact division of nucleus ambiguus; RP, n. raphé pallidus; XII N., hypoglossal nerve tract. $\boldsymbol{B}$, High-power $(63 \times)$ IR-DIC image of RO neurons (see inset in $\boldsymbol{A}$ ) with patch-clamp recording pipette within slice. $\boldsymbol{C}$, Left, Immunoreactivity for $\mathrm{TpOH}$ (Cy5 fluorescence images) was found in three subpopulations: $n$. raphé obscurus (1), n. raphé pallidus (2), and the parapyramidal region (3). Right, Enlargement of box enclosing ventral part of n. raphé obscurus shown on left. $D$, Left, TpOH-immunoreactive neurons in n. raphé obscurus (Cy5 fluorescence). Middle, SP-immunoreactive neurons (Alexa Fluor 488 fluorescence). Right, Merged image showing colocalization of TpOH- and SP-immunoreactive neurons. Arrows indicate the same set of neurons and highlight examples of double-labeled neurons.

neurons in raphé obscurus, some of which colocalize SP. (2) Some individual raphé obscurus 5-HT neurons send collateral branches to both the pre-BötC and the hypoglossal motor nucleus, where a subset of synaptic boutons near respiratory neuron dendrites colocalize 5-HT and SP. (3) Some pre-BötC inspiratory neurons project back to the n. raphé obscurus and impart a respiratory rhythm on 5-HT neuron activity. (4) Inspiratory motor output from both in vitro and in situ preparations is critically dependent on endogenously released 5-HT and SP. (5) In slices this is due to direct actions on pre-BötC neurons. (6) The pharmacology of these effects is consistent with actions on $5-\mathrm{HT}_{2 \mathrm{~A} / 2 \mathrm{C}}$, 5- $\mathrm{HT}_{4}$, and NK-1 receptors. (7) An increase in firing rate of 5-HT neurons augments the frequency of inspiratory motor output in vitro and in situ due to increased activation of $5-\mathrm{HT}_{2 \mathrm{~A} / 2 \mathrm{C}}$ and NK-1 receptors. (8) 5-HT causes some pre-BötC neurons to develop intrinsic bursting behavior. (9) Excitatory effects of 5-HT and SP are due to modulation of multiple neuronal background cation conductances, one of which has properties similar to the newly identified leak $\mathrm{Na}^{+}$channel NALCN, which is critical for normal respiratory rhythm generation ( $\mathrm{Lu}$ et al., 2007). These results provide novel insights into how raphé neurons regulate respiratory network function and have implications for pathophysiological conditions in which abnormal raphé function is proposed to cause instability of breathing.

\section{Materials and Methods}

Experiments were performed with in vitro medullary slice preparations from neonatal rats, which allowed us to combine detailed cellular and network level analyses, and with in situ perfused brainstem-spinal cord preparations from juvenile rats, which generate patterns of cranial and spinal motoneuron activity similar to those in vivo (Paton, 1996; Smith et al., 2007) and provide an experimental bridge to the mature system in vivo. These approaches allowed us to compare results obtained in the neonatal system in vitro to a more intact mature system. All experimental procedures were approved by the National Institute of Neurological Disorders and Stroke Animal Care and Use Committee.

Medullary slice preparations. Procedures for cutting slices were as previously described (Smith et al., 1991; Koshiya and Smith, 1999). Briefly, the medulla of P0-P3 Sprague Dawley rats were dissected in artificial CSF (aCSF) containing the following (in $\mathrm{mM}$ ): $124 \mathrm{NaCl}, 25$ $\mathrm{NaHCO}_{3}, 3 \mathrm{KCl}, 1.5 \mathrm{CaCl}_{2}, 1.0 \mathrm{MgSO}_{4}, 0.5$ $\mathrm{NaH}_{2} \mathrm{PO}_{4}$, and 30 D-glucose, equilibrated with $95 \% \mathrm{O}_{2}$ and $5 \% \mathrm{CO}_{2}(\mathrm{pH} 7.4 \pm 0.05,325 \pm 5$ $\mathrm{mOsm} / \mathrm{L}$ ) at room temperature. Transverse slices (300-400 $\mu \mathrm{m}$ thick) containing the preBötC, the rostral end of the hypoglossal (XII) motor nucleus including XII nerve rootlets, and parts of the medullary raphé nuclei (Fig. 1) were mounted in a recording chamber on a microscope stage and superfused with aCSF, warmed to $27^{\circ} \mathrm{C}$. Rhythmic respiratory activity was maintained by elevating extracellular $\left[\mathrm{K}^{+}\right]$to 8-9 mM.

In situ brainstem-spinal cord preparations. Procedures for setting up the in situ arterially perfused brainstem-spinal cord preparations of juvenile rats were as described previously in detail (Paton, 1996; Smith et al., 2007). These preparations allow independent control of perfusate $\mathrm{O}_{2} / \mathrm{CO}_{2}$ concentrations, as well as administration of pharmacological agents at concentrations that would be incompatible with viability of in vivo preparations. In brief, preheparinized (1000 U, i.p.) male Sprague Dawley rats (70-110 g) were anesthetized deeply with isoflurane until loss of paw withdrawal reflex. Rats were bisected subdiaphragmatically, the head and thorax were immersed in ice-chilled carbogenated Ringer's solution, and the brain was decerebrated precollicularly. The cerebellum was removed to gain direct visual access to the dorsal brainstem surface. Thoracic phrenic (PN) and hypoglossal (XII) nerves (on the left side) were cut distally. Preparations were transferred to a recording chamber and positioned prone, and the head was fixed using ear bars and a snout clamp that ensured the brainstem was orientated similarly in all preparations. A double lumen cannula (DLR-4, Braintree Scientific) was inserted retrogradely into the descending aorta for perfusion. Perfusion was supplied via a peristaltic roller pump (Watson Marlow 505D) and consisted of carbogenated Ringer's solution at $32^{\circ} \mathrm{C}$ (in mM): $125 \mathrm{NaCl}, 24 \mathrm{NaHCO}_{3}$; $3 \mathrm{KCl} ; 2.5 \mathrm{CaCl}_{2} ; 1.25 \mathrm{MgSO}_{4}, 1.25 \mathrm{KH}_{2} \mathrm{PO}_{4}$, and 10 dextrose, $\mathrm{pH} 7.35-$ 7.4 after carbogenation. Osmolality was $290 \pm 5 \mathrm{mOsm} \cdot \mathrm{kg} \mathrm{H}_{2} \mathrm{O}^{-1}$ 
Ficoll $70(1.25 \%)$ was added as an oncotic agent. The second lumen of the cannula was used to monitor aortic perfusion pressure. The baseline perfusate flow was preset between 20 and $24 \mathrm{ml} \cdot \mathrm{min}^{-1}$ and adjusted until the inspiratory motor pattern consisted of an augmenting burst discharge. In addition, vasopressin (200-400 pм as required) was added to the perfusate to raise perfusion pressure to between 80 and $90 \mathrm{mmHg}$ (Pickering and Paton 2006). Vecuronium bromide $\left(4 \mu \mathrm{g} \cdot \mathrm{ml}^{-1}\right.$; Organon Teknica) was added to the perfusion solution to block neuromuscular transmission.

Electrophysiological recordings and data analysis. Inspiratory-related motor discharge in vitro was recorded from XII nerve rootlets with glass suction electrodes (60-90 $\mu \mathrm{m}$ tip diameter) filled with aCSF (Fig. $1 A$ ). In the in situ preparations simultaneous recordings of PN and XII motor nerve activity were obtained with bipolar suction electrodes (100-150 $\mu \mathrm{m}$ tip diameter) mounted on separate three-dimensional micromanipulators. Population recordings in nucleus raphé obscurus in situ, and in some preparations from the pre-BötC, were made with tungsten microelectrodes (1-2 M $\Omega$ ) positioned with a three-axis, motorized micromanipulator (Marzhauser). Population recording sites were marked in some in situ preparations with electrolytic lesions ( $7 \mu \mathrm{A}$ constant current for 10-20 s). All nerve signals were amplified (5000-10,000 times) and bandpass filtered $(0.3-1 \mathrm{kHz}$ ) with a Cyberamp 360 amplifier (Molecular Devices). Raw recordings were rectified and smoothed by analog or digital integration (30-100 ms time constant). Extracellular recordings of single-neuron spike discharges in vitro were obtained with 8-12 $\mathrm{M} \Omega$ glass microelectrodes filled with $0.5 \mathrm{M} \mathrm{Na}$-acetate. Signals were amplified (20,000-70,000 times) and bandpass filtered between 0.1 and $4 \mathrm{kHz}$.

Whole-cell patch-clamp recordings were obtained in vitro with borosilicate electrodes (WPI) (3-4 M 2 ) filled with a solution containing the following (in mM): $125 \mathrm{~K}$-gluconate, $4 \mathrm{NaCl}, 11 \mathrm{EGTA}, 1 \mathrm{CaCl}_{2}, 10$ HEPES, 4 Mg-ATP, 0.3 Na-GTP, 4 Na-phosphocreatine, $\mathrm{pH} 7.3$ adjusted with $\mathrm{KOH}(290 \pm 5 \mathrm{mOsm} / \mathrm{L})$. For measurement of the nonselective cation current (see Fig. 13D), the internal solution contained the following (in mM): $117 \mathrm{CsCl}$, 9 EGTA, 9 HEPES, $1.8 \mathrm{MgCl}_{2}$, 14 Tris-creatinine$\mathrm{PO}_{4}, 4 \mathrm{Mg}$-ATP, and 0.3 Tris-GTP buffered to $\mathrm{pH} 7.3$ with CsOH (290 \pm $5 \mathrm{mOsm} / \mathrm{L})$. Raphé neurons were visualized with infrared-differential interference contrast (IR-DIC) optics (Fig. 1B) (780-900 nm wavelengths, Newvicon extended IR camera and Argus 20 real-time image processor, Hamamatsu Photonics). Whole-cell recordings of XII inspiratory motoneurons were also obtained under IR-DIC visualization up to limits of optical resolution ( $\sim 80 \mu \mathrm{m}$ deep). Recordings from pre-BötC inspiratory neurons were obtained by blind patch-clamp procedures (Smith et al., 1991), which allowed whole-cell recordings from neurons deeper in the slices (up to $150 \mu \mathrm{m}$ from the slice surface). In some in vitro experiments, neurons were filled with biocytin $(0.8-1 \%$ in pipette solution) during whole-cell patch-clamp recordings.

Patch-clamp recordings were made with an EPC-9 or EPC-10 amplifier (HEKA Electronics), low-pass filtered at $3-5 \mathrm{kHz}$, and digitally sampled at $10 \mathrm{kHz}$ with Pulse software (HEKA Electronics). A liquid junction potential of $-10 \mathrm{mV}\left(\mathrm{K}^{+}\right.$-based solution $)$or $-8 \mathrm{mV}\left(\mathrm{Cs}^{+}\right.$-based solution) was corrected off-line. The series resistance was compensated to $85 \%$ using the $2 \mu$ s setting of the amplifier; compensation was extended to the upper limit permitting stability of signals and adequacy of compensation was examined via $5 \mathrm{~ms}$ test pulses.

All extracellular and intracellular signals were also simultaneously acquired digitally ( $10 \mathrm{kHz}$ sampling rates) using a PowerLab A/D converter and Chart v5.0 software (AD Instruments). The parameters describing cellular and network population activity (raphé neuron spiking frequency, XII and PN inspiratory interburst interval/frequency, inspiratory neuron burst frequency, and intraburst spike frequency) were calculated off-line using Chart v5.0 and Igor Pro (Wavemetrics) software with custom automated analysis procedures and hand checked for accuracy. Interburst intervals were calculated from integrated (50 ms integration time constant) inspiratory discharge or single-neuron inspiratory bursts as the average interval between the onset times of consecutive bursts (10-30 bursts) and then used to calculate burst frequency.

Whole-cell currents were analyzed with Pulsefit (HEKA Electronics), Chart, and Igor Pro software. The leak conductance was determined by linear regression analysis applied to the linear region of slow voltage- clamp ramps $(30 \mathrm{mV} / \mathrm{s})$. Neurons with evidence of poor space clamp, such as unclamped action potential currents during the voltage-clamp ramps, were excluded from analysis.

Statistical significance was determined by a two-tail Student's $t$ test for unpaired or paired data (when $n \geq 10$; data presented as mean \pm SEM), or by a nonparametric Wilcoxon signed-rank test (when $n<10$; data presented as the median value). ANOVA followed by a Newman-Keuls posttest (Prism 4, GraphPad Software) was also used where appropriate.

Pharmacological procedures and solutions. In slice preparations, each drug was applied only once in a given slice, and only one slice was obtained per animal. Drugs were either bath applied or continuously microinfused into specific areas, as indicated. Microinfusion pipettes (10-15 $\mu \mathrm{m}$ inner diameter) were placed either into the raphé obscurus or into the pre-BötC visualized with IR-DIC optics (Fig. $1 A$ ). In the arterially perfused preparations, drugs were delivered systemically via the perfusate. In some in situ preparations, microinfusion pipettes were placed into raphé obscurus while simultaneously recording neuron activity with a tungsten electrode attached to the outer surface of the perfusion pipette. Drug solutions were ejected by applying low positive pressure $(20-30 \mathrm{mmHg})$ with a precision pressure control and valve system (Picospritzer-IID, General Valve Corp, and 2PK + Pressure Controller, ALA Scientific Instruments).

For in vitro experiments, methysergide maleate (MeSG) and ketanserin tartrate (KET) (Sigma-RBI) were dissolved in 10\% DMSO to make a $10 \mathrm{~mm}$ stock and diluted with aCSF immediately before use. The maximum final concentration of DMSO was $0.01 \%$. (S)-1-(2\{3-\{3,4Dichlorophenyl $\}$-1-\{3-isopropoxyphenylacetyl $\}$ piperidin-3-yl $\}$ ethyl)-4phenyl-1-azabicylo (2.2.2) octane chloride [SR 140333 (SR)] (a gift from Sanofi-Aventis) was dissolved in $7 \%$ ethanol to make a $1 \mathrm{~mm}$ stock and then diluted with aCSF immediately before use. The maximum final concentration of ethanol was $0.07 \%$. $\alpha$-Phenyl-1-(2-phenylethyl)-4piperidinemethanol [MDL 11,939 (MDL)] (Tocris Bioscience) was dissolved in ethanol (maximum final concentration of $0.1 \%$ ethanol). 8- [5(2,4-Dimethoxy-5-(4-trifluoromethylphenylsulfonamido)phenyl)-5oxopentyl]-1,3,8-triazaspiro[4.5] decane-2,4-dione hydrochloride [RS 102221 hydrochloride (RS) ] and 1-methyl- $1 \mathrm{H}$-indole-3-carboxylic acid, [1-[2-[(methylsulfonyl)amino]ethyl]-4-piperidinyl]methyl ester [GR 113808 (GR)] (Tocris Bioscience) were dissolved in DMSO (maximum final concentration of $0.1 \%$ DMSO). Serotonin $(30 \mu \mathrm{M})$ and [Sar ${ }^{9}$ $\operatorname{Met}\left(\mathrm{O}_{2}\right)^{11}$ ]-substance $\mathrm{P}(1 \mu \mathrm{M})$ were dissolved in aCSF.

For in situ experiments, MeSG and SR 140333 were dissolved as indicated above but in the Ringer's solution used for perfusion. MDL 11,939 (Tocris Bioscience) was dissolved in $100 \%$ ethanol and then diluted in the perfusate solution (maximum final concentration of $0.1 \%$ ethanol). RS 102221 hydrochloride and GR 113808 (Tocris Bioscience) were dissolved in DMSO (maximum final concentration of $0.1 \%$ DMSO).

Neurons in the raphé nuclei were stimulated by local microinfusion (Al-Zubaidy et al., 1996) of either of the following: (1) AMPA (5 $\mu \mathrm{M}$, dissolved in aCSF with $\left[\mathrm{K}^{+}\right]=3 \mathrm{~mm}$ in vitro or in Ringer's solution for in situ experiments) or (2) aCSF with $\left[\mathrm{K}^{+}\right]$elevated to $15 \mathrm{~mm}$, used for in vitro experiments in which monosynaptic projections were studied after blocking glutamate receptors with CNQX. With stimulation of raphé neurons by AMPA, the concentration of microinfused AMPA used was chosen as that which increased the spiking frequency of individual raphé neurons over the natural spiking frequency range observed for 5-HT neurons in vivo (Mason, 1997). In some in vitro experiments, sodium currents were blocked with tetrodotoxin (TTX, $1 \mu \mathrm{M}$ ). Potassium and calcium currents were blocked with tetraethylammonium (TEA) chloride $(20 \mathrm{~mm})$ and $\mathrm{CdCl}_{2}(200 \mu \mathrm{M})$, respectively. Non-NMDA glutamate receptors were blocked with CNQX (6-cyano-7-nitroquinoxaline-2,3dione; $20 \mu \mathrm{M}$ ) (Koshiya and Smith, 1999). Vesicular neurotransmitter release was blocked with $\mathrm{CdCl}_{2}(200 \mu \mathrm{M})$. All reagents were purchased from Sigma-RBI.

Neuroanatomy and immunohistochemistry. Neonatal slices were fixed in $4 \%$ paraformaldehyde in $0.1 \mathrm{~m}$ phosphate buffer for $\sim 24 \mathrm{~h}$ at $4^{\circ} \mathrm{C}$, cryoprotected at $4^{\circ} \mathrm{C}$ in $30 \%$ sucrose, and for immunohistochemistry coronally sectioned (50 $\mu \mathrm{m}$ thick) on a freezing microtome. All sections were preincubated for $1 \mathrm{~h}$ with 10\% donkey serum in PBS with Triton $\mathrm{X}-100(0.5 \%)$ and subsequently incubated for $24-48 \mathrm{~h}$ at $4^{\circ} \mathrm{C}$ with pri- 
mary antibodies: mouse anti-tryptophan hydroxylase $(\mathrm{TpOH})$ (the ratelimiting enzyme for 5-HT biosynthesis) (1:500; Sigma), goat antiserotonin (1:500; Immunostar), and/or rabbit anti-substance P (1:500; Immunostar). Floating sections were incubated for $2 \mathrm{~h}$ at room temperature with a mixture of donkey anti-mouse IgG conjugated with Cy5 or Cy3 (1:500; Jackson ImmunoResearch) and/or donkey anti-rabbit IgG conjugated with Alexa 488 (1:500; Invitrogen) or donkey anti-goat IgG conjugated with $\mathrm{Cy} 3$. For fluorescence labeling of neurons filled with biocytin during electrophysiological recording, slices were incubated with Texas Red-Avidin D or AMCA-Avidin D (Vector Laboratories). Tissue was mounted on slides and covered with antifading medium (Prolong Gold, Invitrogen). Confocal images were obtained with a laserscanning microscope (LSM 510 Meta, Zeiss). AMCA, Alexa Fluor 488, Cy3, and Texas Red or Cy5 were detected at 405, 488, 543, and $633 \mathrm{~nm}$ emission wavelengths, respectively. Images were acquired and processed with Zeiss LSM 510 system software and presented as two-dimensional projections from serial optical sections ( $Z$-stacks) or in some cases single optical sections $(0.3 \mu \mathrm{m})$

For some experiments we reconstructed biocytin-filled neurons and axonal projections in whole mounts of the in vitro slices. Slices were fixed and rinsed as described above, then pretreated for $30 \mathrm{~min}$ with $0.3 \%$ $\mathrm{H}_{2} \mathrm{O}_{2}$ in PBS with $0.5 \%$ Triton X-100. Slices were then incubated overnight at $4^{\circ} \mathrm{C}$ in an avidin-biotinylated horseradish peroxidase reagent (Vectastain, Vector Laboratories), rinsed, and developed with nickelintensified DAB (DAB kit, Vector Laboratories). Slices were dehydrated, cleared with methylsalicylate, mounted in well slides, coverslipped, and coated with Permount (Fisher Scientific). Labeled cell bodies, dendritic fields, and axons were visualized with light microscopy. Reconstructions of cell morphology and axonal projections were obtained from photomicrographs of multiple focal planes, composited as a single twodimensional projection image, and then traced in Photoshop 7.0 (Adobe Systems).

For verification of recording sites marked by electrolytic lesions in the in situ preparations, the brainstem was removed at the conclusion of the experiment and immersed in phosphate-buffered $4 \%$ paraformaldehyde at $4^{\circ} \mathrm{C}$ for at least $24 \mathrm{~h}$. The brainstem was then sectioned coronally (50 $\mu \mathrm{m})$ with a freezing microtome. After wet mounting the slices on glass slides, slices were counterstained with cresyl violet for cytoarchitectonic identification of lesion sites.

\section{Results}

\section{Rhythmic slices contain 5-HT neurons that colocalize SP}

The brainstem raphé system has a multinuclear organization (Jacobs and Azmitia, 1992), and the morphological connections of specific subpopulations to the respiratory network have not been well established. Accordingly, to identify the locations of specific subpopulations for subsequent electrophysiological recording and manipulation of cell activity, we mapped the spatial distribution of serotonergic neurons in slice preparations that generated respiratory output (Fig. 1A) using immunohistochemistry for $\mathrm{TpOH}$ ( $n=12$ slices) and/or 5-HT ( $n=9$ slices). We also investigated axonal projections from individual raphé neurons to the pre-BötC and XII motor nucleus at the level of our slice preparations (below). TpOH-immunoreactive neurons at this level of the medulla were located in three subpopulations: the midline $\mathrm{n}$. raphé obscurus and $n$. raphé pallidus, and the bilateral parapyramidal region in the ventrolateral medulla (Fig. $1 C$ ). TpOH immunolabeled cells were double labeled by 5 -HT immunostaining (data not shown). Previous reports have demonstrated SP in cell bodies of raphé neurons (Menetrey and Basbaum, 1987; Dean et al., 1993). By double immunostaining, we confirmed the colocalization of TpOH and SP immunoreactivity within n. raphé obscurus (Fig. 1D) and the other subnuclei.

We focused our analysis on raphé obscurus, since these cells have been implicated in control of breathing (Al-Zubaidy et al., 1996; Feldman et al., 2003), and they form a relatively compact bilateral cluster along the midline that could be readily visualized in vitro (Fig. $1 A, B$ ) and could be easily located along the midline in the in situ perfused brainstem-spinal cord preparations for electrophysiological recording and neuronal activation. This facilitated our comparative analysis of activity patterns and modulation of respiratory network activity by this neuronal group in our two experimental preparations. Moreover, this subpopulation of raphé cells was of particular interest because, as demonstrated below, these cells exhibited rhythmic modulation of spiking activity indicative of interactions with the respiratory network, and we found that activation of this population caused strong excitation of the respiratory network. We have not yet systematically analyzed whether activation of the parapyramidal or pallidal raphé cell groups (Fig. 1C) can also drive the respiratory network.

\section{Spontaneous activity of raphé obscurus 5-HT neurons in vitro} 5-HT neurons in vivo generate low-frequency regular tonic spiking activity that has been proposed to be a fundamental electrophysiological attribute (Jacobs and Azmitia, 1992). 5-HT neurons exhibit similar activity in brain slices (Vandermaelen and Aghajanian, 1983) and in culture (Wang et al., 2001). Similarly, we determined that in our slice preparations, most neurons in the n. raphé obscurus [examined by either whole-cell $(n=30)$ or extracellular $(n=25)$ recording] spontaneously generated action potentials at a low frequency, under both normal extracellular $\mathrm{K}^{+}$concentration $\left(\left[\mathrm{K}^{+}\right]_{\mathrm{o}}\right)(3 \mathrm{~mm}$; spike frequency $f=1.1 \pm 0.51$ $\mathrm{Hz} ; n=15$ ) (Fig. $2 A$, gray trace) and elevated $\left[\mathrm{K}^{+}\right]_{\mathrm{o}}$, as typically used in our experiments $(8-9 \mathrm{~mm}, f=1.4 \pm 0.32 \mathrm{~Hz}$; mean $\pm \mathrm{SD}$; $n=40$ ) (Fig. $2 A$, black trace). This spontaneous activity has not been previously shown in slice preparations generating rhythmic respiratory output. The spontaneous firing pattern was regular, typical of 5-HT neurons (Vandermaelen and Aghajanian, 1983; Jacobs and Azmitia, 1992; Bayliss et al., 1995; Wang et al., 2001). Action potentials had a relatively long duration $(2.4 \pm 0.35 \mathrm{~ms}$ measured at half-height; $n=10$ neurons), a slight shoulder on the repolarizing phase, and were followed by a pronounced afterhyperpolarization (AHP) (Fig. $2 \mathrm{~B}$ ). Elevated $\left[\mathrm{K}^{+}\right]_{\mathrm{o}}$ reduced the AHP but did not disrupt the spontaneous spiking, allowing us to conduct the experiments under these conditions, which are optimal for maintaining inspiratory rhythm generation in our slice preparation.

We also characterized the spike frequency versus applied current $(f-I)$ relations to determine whether the spiking behavior in our slice preparation matched that observed in other experimental preparations. Depolarizing current steps caused an initial rise in firing frequency, followed by adaptation, in agreement with previous slice and cultured neuron experiments (Bayliss et al., 1995; Wang et al., 2001). The initial peak and the steady-state firing frequencies are linear functions of the injected current (Fig. 2C) similar to those reported previously (Wang et al., 2001), with steady-state $f-I$ gain of $0.1 \pm 0.02 \mathrm{~Hz} / \mathrm{pA}(n=22)$.

Next, we verified that raphé neurons with regular spiking pattern and prominent AHP were 5-HT neurons. Patch-clamped raphé neurons were filled with biocytin (Fig. 2D, left) and then processed for $\mathrm{TpOH}$ immunohistochemistry (Fig. $2 \mathrm{D}$, middle). We found that all biocytin-filled neurons $(n=15)$ exhibited a regular firing pattern and were also immunoreactive for $\mathrm{TpOH}$ (Fig. $2 D$, right). We conclude that the electrophysiological criteria used to identify raphé neurons in subsequent experiments (i.e., tonic regular firing pattern at $1-2 \mathrm{~Hz}$ and prominent $\mathrm{AHP}$ ) are accurate predictors for 5-HT-containing neurons, as has been 
shown previously in vitro and in vivo (Mason, 1997; Wang et al., 2001).

Raphé obscurus neurons are reciprocally connected to neurons in the respiratory network

We obtained evidence that a subset of neurons in n. raphé obscurus receives excitatory input from the respiratory network. Thus, $47 \%(n=37)$ of regularly spiking raphé neurons showed an increase in firing rate during the inspiratory phase of the respiratory cycle (Fig. $3 A, B$ ). Hyperpolarizing neurons to $-60 \mathrm{mV}$ eliminated spontaneous tonic firing, and revealed the presence of synaptic input synchronous with inspiratory motor output (Fig. $3 C, D)$. We note that the actual percentage of raphé obscurus neurons that receive inspiratory synaptic drive is likely higher, as some axonal fibers would likely be sectioned in our slice preparations. Firing of some raphé neurons in phase with inspiratory output in vivo has previously been reported (Lindsey et al., 1992), although the neurochemical content of those neurons was not determined. The functional significance of these feedback connections to raphé neurons, presumably from pre-BötC neurons (see below), and the associated phasic augmentation of raphé neuron activity is not yet clear, but burst firing could enhance the release of colocalized neuropeptides (Iverfeldt et al., 1989).

We analyzed the morphology and axonal projections of a group of raphé obscurus 5-HT neurons $(n=8)$ after wholecell recording and neuronal staining with biocytin. Figure $3 E$ summarizes the main morphological features and projection patterns for these neurons. Neurons with somata located in the n. raphé obscurus (e.g., blue- and red-colored cells) had axons projecting laterally either in the direction of $(n=6)$ or within the pre-BötC $(n=2)$, as identified by recording from and biocytin staining of pre-BötC inspiratory neurons in the same slices (Fig. 3E). Some of these raphé neurons also sent collateral axons in the dorsal direction toward hypoglossal motoneurons (e.g., red-colored cell). All of these labeled neurons were also serotonergic, as indicated by $\mathrm{TpOH}$ immunoreactivity.

Consistent with these results, we found the pre-BötC region to be densely innervated with serotonergic and SP-containing fibers (Fig. 4A). We also established that some of these fibers, as well as a subset of presynaptic boutons on some dendrites of electrophysiologically identified pre-BötC inspiratory neurons, colocalize 5-HT and SP (Fig. 4B-D). Similarly, we found that many $\mathrm{TpOH} / 5-\mathrm{HT}$ immunostained fibers project dorsally along or near the midline toward the XII motor nucleus, and some dendrites of electrophysiologically identified inspiratory XII motoneurons are associated with double-labeled presynaptic boutons (supplemental Fig. 1, available at www.jneurosci.org as supplemental material). We also found that many pre-BötC neurons $(n=14 / 18)$ send axons through the $\mathrm{n}$. raphé obscurus. Two of these cells had axon collateral branches in this nucleus (e.g., black neuron in Fig. 3E), which could mediate the rhythmic synaptic drive we observed in raphé obscurus cells synchronous with inspiratory motor output (Fig. $3 A-D$ ).

Based on these findings, we conclude that tonically firing neurons in the n. raphé obscurus containing 5-HT and SP project to pre-BötC and XII neurons, and many raphé 5-HT neurons receive reciprocal projections from pre-BötC inspiratory neurons.

\section{Generation of respiratory motor output in vitro and in situ depends on endogenous 5-HT and SP released by raphé neurons}

We next investigated whether endogenously released 5-HT and SP modulate baseline inspiratory network activity in our slice and in situ perfused preparations. To obtain detailed dose-response relationships in slices, receptor antagonists were either microinfused directly into the pre-BötC or bath applied. For in situ preparations, the antagonists were delivered systemically via the perfusate. 5-HT receptors were blocked with the following: (1) MeSG, which is a nonselective $5-\mathrm{HT}$ receptor $\left(5-\mathrm{HT}_{1}\right.$ and $\left.5-\mathrm{HT}_{2}\right)$ 

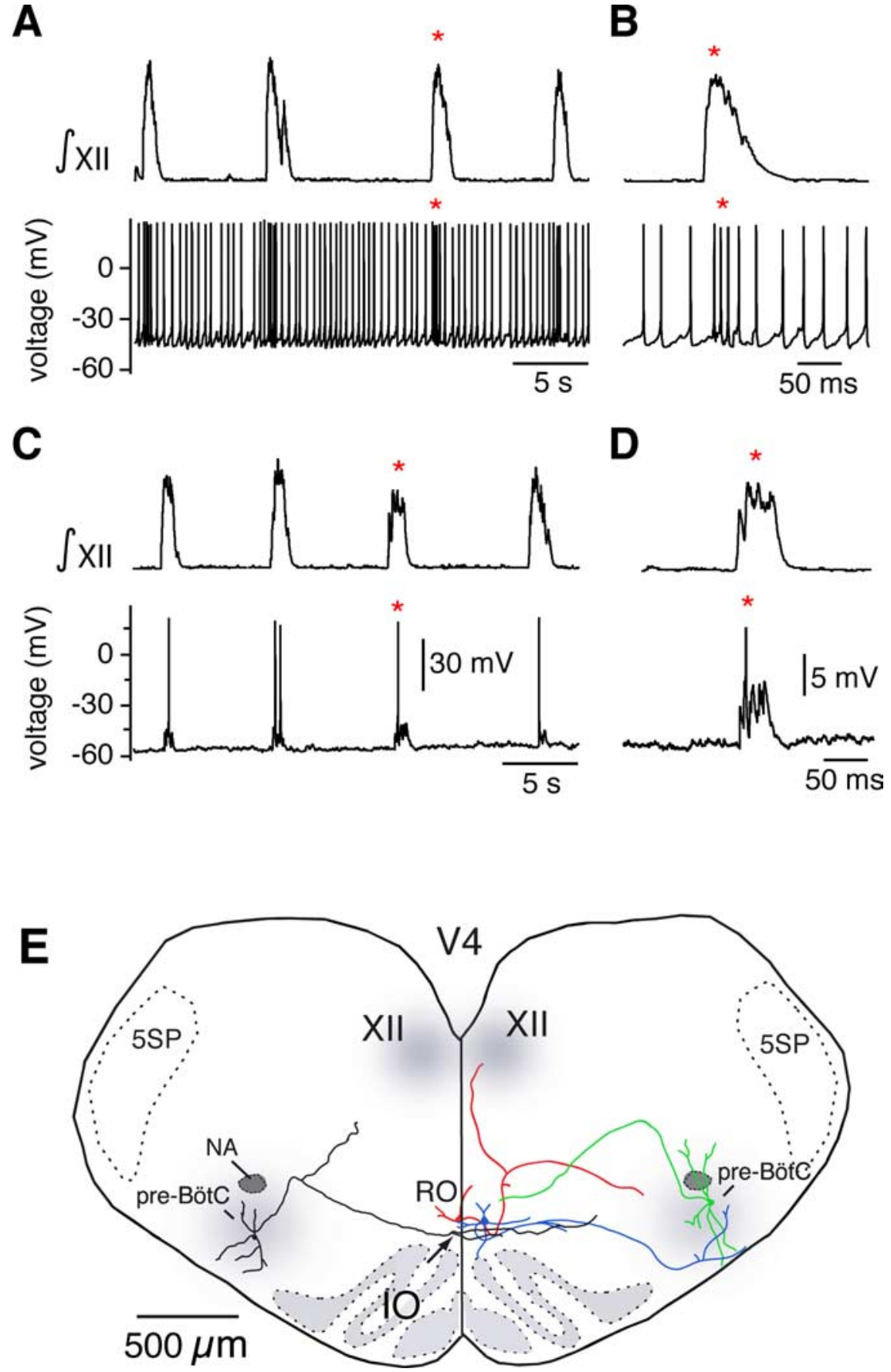

Figure 3. Then. raphé obscurus is interconnected with the pre-BötC and the XII motor nucleus. A, Simultaneous recording from the XII nerve ( $\int X I I$, top) and from a neuron in the n. raphe obscurus (bottom), showing that some raphé neurons increase their spike rate during inspiratory network activity. $\boldsymbol{B}$, Expanded view of augmented neuronal spiking during inspiratory activity (from $\boldsymbol{A}$ indicated by $\left.{ }^{*}\right)$. $\boldsymbol{C}$, Injecting hyperpolarizing current $(20 \mathrm{pA})$ into the raphé neuron to prevent spontaneous firing reveals excitatory depolarizing synaptic potentials phase locked to XII motor output. D, Expanded view of depolarizing potential during inspiratory activity (indicated by ${ }^{*}$ ). $\boldsymbol{E}$, Composite reconstruction of two biocytin-filled raphé obscurus (RO) neurons and two pre-BötC inspiratory neurons illustrating patterns of axonal projections. A pre-BötC inspiratory neuron (green) with axonal projection toward RO and a RO neuron (blue) with projection into pre-BötC were both labeled in the same slice. Other neurons (RO red, pre-BötC black) were labeled and reconstructed in individual slices and rescaled so that all neurons could be composited to represent different spatial patterns of synaptic projections. This other RO neuron projected to hypoglossal (XII) motor nucleus with collateral projections toward pre-BötC. The other pre-BötC neuron (left, black) projected through RO with collateral branching and arborizations in this region. 5SP, Spinal trigeminal nucleus.

antagonist but is also a weak agonist at $5-\mathrm{HT}_{1 \mathrm{~A} / \mathrm{B} / \mathrm{D}}$ receptors; (2) KET, which is selective for $5-\mathrm{HT}_{2 \mathrm{~A}}$ and $5-\mathrm{HT}_{2 \mathrm{C}}$ receptors, but also interacts with $\alpha$-adrenergic receptors; (3) MDL, which is highly selective for $5-\mathrm{HT}_{2 \mathrm{~A}}$ receptors, with 300 -fold selectivity for
$5-\mathrm{HT}_{2 \mathrm{~A}}$ over $5-\mathrm{HT}_{2 \mathrm{C}}$; (4) RS, which exhibits 100 -fold selectivity for $5-\mathrm{HT}_{2 \mathrm{C}}$ over $5-\mathrm{HT}_{2 \mathrm{~A}}$ receptors; and (5) GR, with 300fold selectivity for $5-\mathrm{HT}_{4}$ over $5-\mathrm{HT}_{1 \mathrm{~A} / 2 \mathrm{~A} / 2 \mathrm{C}}$.

Bath-applied ketanserin has previously been found to attenuate baseline inspiratory rhythmic activity in vitro in slices from neonatal mice (Peña and Ramirez, 2002), implicating involvement of $5-\mathrm{HT}_{2 \mathrm{~A}}$ receptors. We sought to first confirm this finding and then extend it by using more selective antagonists, as well as to use focal drug application to determine whether endogenously released 5-HT is acting directly on pre-BötC neurons. We also extended the analysis to determine whether activation of other 5-HT receptor subtypes is required for baseline inspiratory activity in vitro, including 5- $\mathrm{HT}_{4}$ (Manzke et al., 2003) and $\mathrm{HT}_{2 \mathrm{C}}$ receptors. Finally, we examined the role of 5-HT receptor subtypes in generation of inspiratory motor output in the intact juvenile rat nervous system, because recent experimental observations in situ (Toppin et al., 2007) have led to different results than those obtained in neonatal mouse slices. To examine the role of endogenously released SP, we used SR to block activation of NK-1 receptors, because these receptors are densely distributed within the pre-BötC (Gray et al., 2001; Wang et al., 2001; Stornetta et al., 2003) and influence respiratory activity, particularly in the neonatal system (Gray et al., 2001; Feldman et al., 2003; Morgado-Valle and Feldman, 2004; Hayes and Del Negro, 2007). The experimental design we used is novel in that our approach (1) extends previous work in slices to include newer, more selective antagonists, (2) determines the relevance to the intact brain by extending the experiments to in situ preparations, and (3) compares the effect of blocking 5-HT and NK-1 receptors alone with the effect of blocking both together.

In vitro slices

Bath application of MeSG $(n=9)$, KET $(n=8)$, or MDL $(n=6)$ progressively reduced inspiratory burst frequency and amplitude of integrated XII motor discharge in a concentration-dependent manner, nearly completely blocking generation of inspiratory activity at the higher concentrations (Fig. 5). The 5- $\mathrm{HT}_{2 \mathrm{C}}$ (RS, $n=7)$ and $5-\mathrm{HT}_{4}(\mathrm{GR}, n=3)$ receptor antagonists were less effective, but also significantly reduced inspiratory burst frequency to $55 \pm 13 \%(p<0.05)$ of control for RS, and $75 \pm 20 \%$ of control ( $p<0.05$ ) for GR, while simultaneously reducing XII burst amplitude to $84 \pm 28 \%$ of control for RS, and $88 \pm 14 \%$ of 
control $(p>0.05)$ for GR at the highest concentrations applied. The NK-1 receptor antagonist SR potently reduced the frequency and amplitude of XII inspiratory motor discharge in a concentrationdependent manner, nearly completely blocking generation of inspiratory activity at the highest dose $(10 \mu \mathrm{M}, n=7)$. This result indicates that $\mathrm{SP}$ is released endogenously even under baseline conditions when 5-HT neurons are firing at a low rate, consistent with previous biochemical measurements of SP release in response to electrical stimulation (Iverfeldt et al., 1989).

Based on the dose-response relationships defined above, we bilaterally microinfused MeSG, KET, or SR into the preBötC of slices (see Fig. $1 \mathrm{~A}$ for pipette configuration), at the $\mathrm{IC}_{50}$ concentrations, to directly determine the effect of 5-HT receptor activation on the pre-BötC rhythm-generating network. MeSG (5 $\mu \mathrm{M})$ reduced inspiratory burst frequency in the XII nerve by $79.5 \pm 4.3 \%(p<$ $0.001 ; n=8$ ) (Fig. $6 A$ ) after 2 min of bilateral microinfusion into the pre-BötC. Similarly, KET $(7.5 \mu \mathrm{M})$ reduced the frequency by $78.3 \pm 5.7 \%(p<0.001 ; n=7)$ (Fig. 6B), while SR $140333(7.5 \mu \mathrm{M})$ reduced frequency by $79.3 \pm 4.9 \%(p<$ $0.001 ; n=7$ ) (Fig. 6C).

We then microinfused 5-HT or SarMet SP (an NK-1 receptor agonist) directly into the pre-BötC, bilaterally to verify that the overall actions of 5-HT and SP on inspiratory activity are excitatory at this level as implied by the data using antagonists. Both agonists had strong and relatively fast excitatory effects on inspiratory output (Fig. $6 D-F)$. Serotonin $(30 \mu \mathrm{M})$ increased inspiratory discharge frequency by $107 \pm 7.9 \%(p<0.001 ; n=5)$ (Fig. $6 D, E)$ and reduced the amplitude of inspiratory bursts by $23 \pm 5.7 \%(p<0.001 ; n=5)$ (Fig. 6D). Similarly, Sar-Met SP (1 $\mu \mathrm{M})$ increased the frequency by $115 \pm 8.2 \%$ $(p<0.001 ; n=5)$ (Fig. $6 F$ ) and reduced the amplitude of inspiratory bursts by $21 \pm 4.7 \%(p<0.001 ; n=5$; data not shown).

Together these results indicate that 5-HT and SP are released from 5-HT neurons of the $n$. raphe obscurus under baseline conditions. These neurotransmitters act on $5-\mathrm{HT}_{2}, 5-\mathrm{HT}_{4}$, and $\mathrm{NK}-1$ receptors to provide tonic excitation of neurons in both the pre-BötC and XII motoneuron pools. This input is critical for spontaneous generation of respiratory output from the medullary slice preparation. It has previously been shown that focal microinfusion of kainic acid into the midline raphé in medullary brain slices causes disruption of respiratory output, suggesting that this region is the source of en-
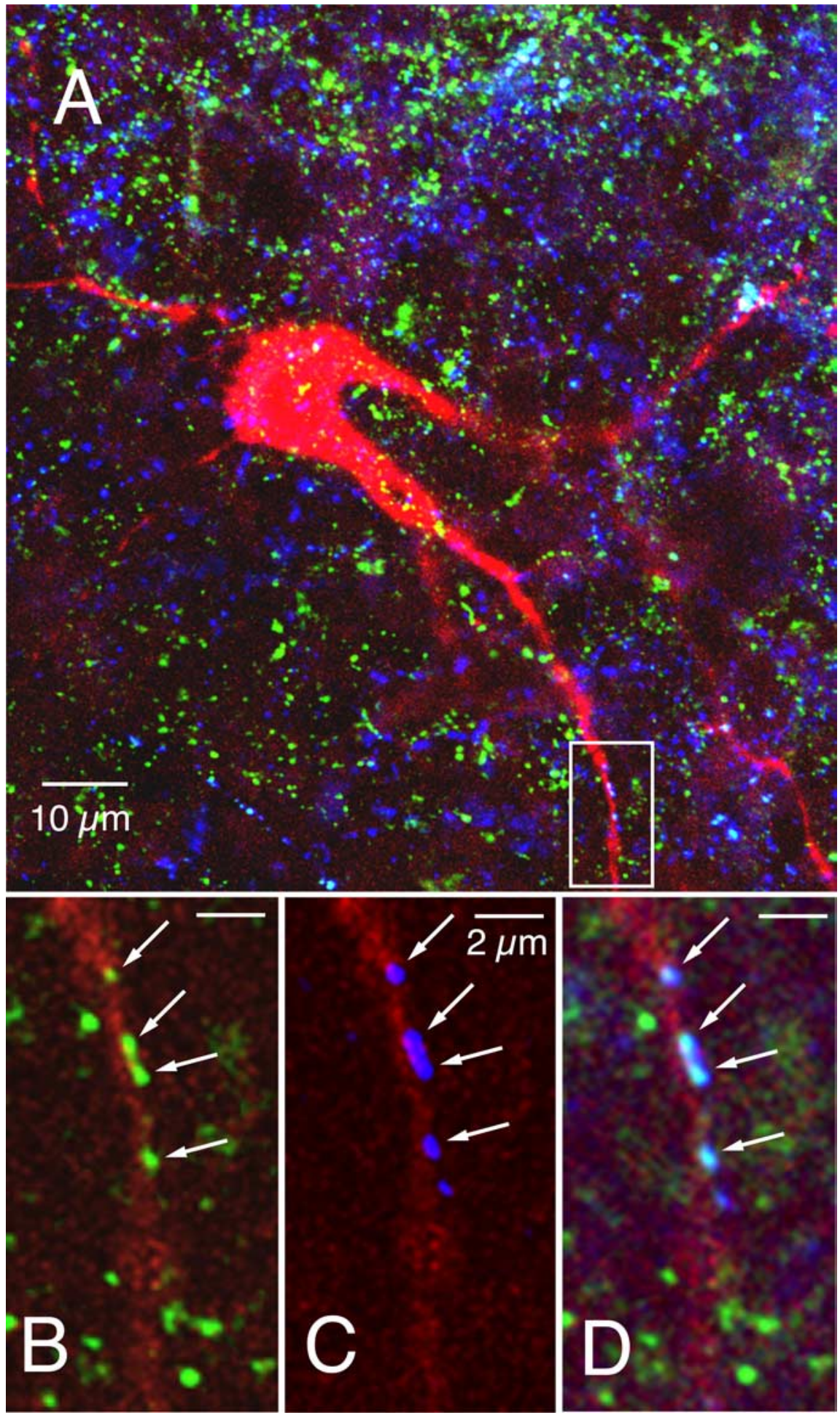

Figure 4. The pre-BötC region is innervated by serotonergic and SP-containing fibers, and a subset of presynaptic boutons on inspiratory neurons colocalize 5-HT and SP. A, Confocal image showing simultaneous labeling of an inspiratory pre-BötC neuron (red, AMCAAvidin D) surrounded by fibers immunoreactive for 5-HT (blue, Cy3) and SP (green, Alexa Fluor 488) (63× magnification, 2-dimensional projection from merged image stack). $\boldsymbol{B}-\boldsymbol{D}$, Magnification of area outlined by white box in $\boldsymbol{A}$ showing examples of $S P(\boldsymbol{B}), 5-\mathrm{HT}(\boldsymbol{C})$, and colocalization of 5-HT and SP ( $\boldsymbol{D}$, merged images) in presynaptic boutons (arrows) on a dendrite of an electrophysiologically identified inspiratory neuron. Images are from a single scan plane $(63 \times, 0.3 \mu \mathrm{m}$ optical section thickness).

dogenous 5-HT that provides tonic drive to the respiratory network (Peever et al., 2001). Consistent with this, we found that spontaneous activity of raphé obscurus neurons was critical for production of rhythmic inspiratory network activity. We microinfused aCSF solution with zero $\left[\mathrm{K}^{+}\right]$into the $\mathrm{n}$. raphé obscurus 

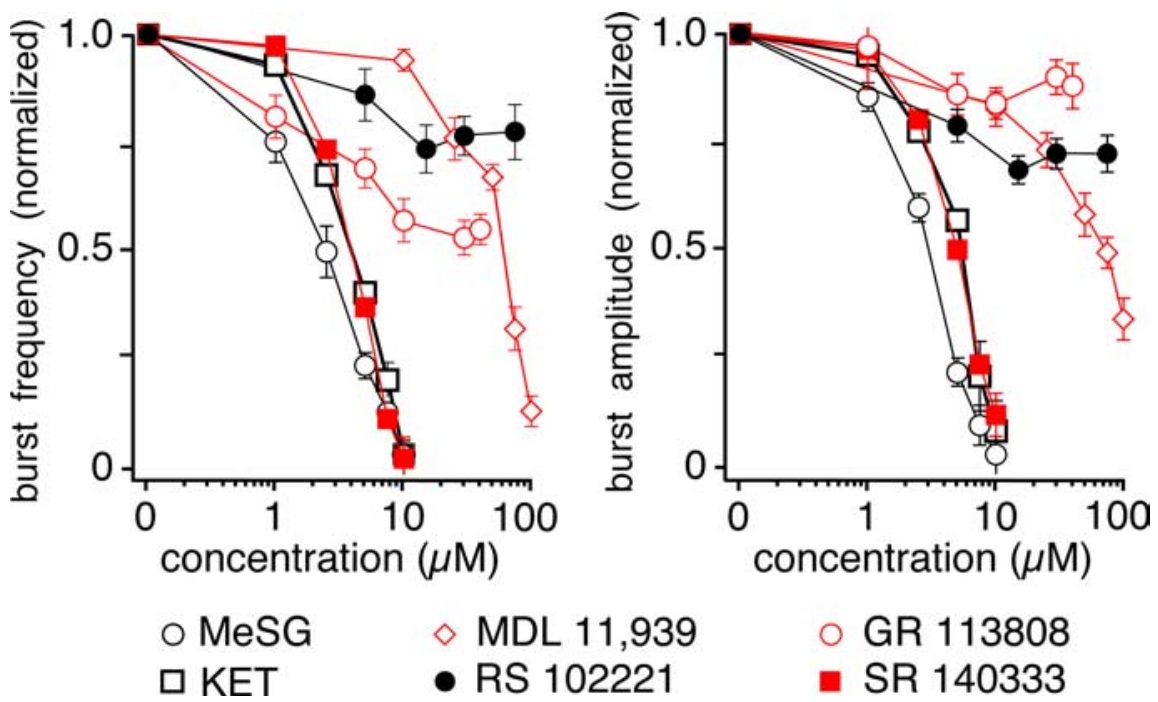

\section{o GR 113808 \\ - SR 140333}

Figure 5. Inspiratory motor output in the rhythmic slice in vitro depends on endogenous activation of 5-HT $2 \mathrm{~A}$ and NK-1 receptors. Bath application of methysergide (MeSG, $n=9$ ), ketanserin (KET, $n=8), \operatorname{MDL} 11,939(n=6), \operatorname{RS} 102221(n=7), \mathrm{GR}$ 113808 ( $n=3)$, and SR 140333 ( $n=7$ ) produced a concentration-dependent depression of baseline inspiratory activity (monitored by recordings of integrated XII motoneuron population activity), with a reversible reduction in the inspiratory XII burst frequency (left) and burst amplitude (right). Panels show steady-state dose-response relations normalized to control. Amplitudes are measured from the peak of the integrated XII population activity. Data points represent mean values \pm SEM.

To determine whether generation of inspiratory activity in situ is also dependent on endogenous SP, SR was applied systemically via the perfusate. This caused a statistically insignificant increase of inspiratory burst frequency ( $p=0.84$ at 100 $\mu \mathrm{M}$ relative to control, $n=3$ ) (Fig. $8 A$ ). The amplitude of inspiratory motor output decreased significantly $(p<0.01, n=$ 3 ) and did so at lower SR concentrations than those that were required to decrease the amplitude of integrated pre-BötC population activity ( $p<0.05, n=3$ ) (Fig. $8 B$ ). This suggests that endogenously released SP stimulates both the pre-BötC and motor output pathways, but may have its greatest effect downstream from preBötC inspiratory burst generation.

We then applied the nonspecific 5-HT receptor antagonist MeSG followed by the NK-1 receptor antagonist SR to the perfused preparation. MeSG $(20 \mu \mathrm{M})$ reduced $\mathrm{PN}$ and XII burst amplitudes to $37.06 \pm$ 20.91\% $(p<0.05, n=3)$ and $40.13 \pm$ $16.20 \%(p<0.05, n=3)$ of control, respectively, while not significantly affecting inspiratory burst frequency $(p=0.34, n=$

of brain slices while performing whole-cell or extracellular recordings from raphé neurons. We found that this caused cessation of spontaneous action potential generation from raphé neurons and rhythmic inspiratory output stopped within $1 \mathrm{~min}$ from the start of microinfusion ( $n=5$ slices; data not shown).

In situ preparations

The $5-\mathrm{HT}_{2 \mathrm{~A}}$ and $5-\mathrm{HT}_{4}$ receptor subtype-specific antagonists $\mathrm{MDL}$ and GR produced large perturbations of inspiratory motor output in situ. Motor output was recorded from phrenic (PN) and XII nerves while simultaneously recording pre-BötC population activity. Application via the perfusate of increasing concentrations of MDL did not significantly alter inspiratory burst frequency ( $p=0.98, n=8)$ over the antagonist concentration range tested but progressively reduced the amplitude of integrated PN and XII motor outputs $(p<0.0001$ at the highest concentration relative to control, $n=8)$ as well as pre-BötC inspiratory bursts $(p<0.05, n=8)$ (Fig. 7). Motor output was completely eliminated in six of eight preparations by MDL at the highest concentration applied $(50 \mu \mathrm{M})$. In contrast, the $5-\mathrm{HT}_{2 \mathrm{C}^{-}}$ specific antagonist RS significantly decreased the frequency (to $61.0 \pm 8.1 \%$ of control at $50 \mu \mathrm{M}, p<0.05, n=4)$ as well as the amplitude of inspiratory motor output (to $36 \pm 8.7 \%, p<0.01$, $n=4)$ and pre-BötC population activity $(16.0 \pm 10.2 \%$ of control, $p<0.05)$. Together, these data suggest that the frequency and amplitude of inspiratory activity may be differentially affected by different $5-\mathrm{HT}_{2}$ receptor subtypes in the intact system. Results with the application of the $5-\mathrm{HT}_{4}$ receptor antagonist GR were similar to those with MDL, although slightly less potent. Inspiratory (PN) burst amplitudes decreased (to 38\% of control, $p<0.05, n=3$ ), and burst frequency was also reduced (to $76 \%$ of control at $80 \mu \mathrm{M}, n=3$ ). Compared with MDL, GR produced a larger decrease in PN burst amplitude than XII amplitude (Fig. $7 B, C$ ), suggesting differential 5-HT receptor subtype-specific modulation of spinal versus cranial inspiratory motor output.
3) (Fig. 9). Adding SR $(20 \mu \mathrm{M})$ to MeSG significantly reduced inspiratory burst frequency (to $13.92 \pm 10.2 \%$ of control, $p<$ $0.01, n=3$ ) and further decreased motor output amplitudes (PN, $13.16 \pm 9.6 \%$ of control, $p<0.05, n=3$; XII, $5.72 \pm 4.2 \%$ of control, $p<0.01, n=3$ ), ultimately eliminating motor output completely in two of the three preparations tested.

\section{Increased raphé neuronal activity excites the respiratory network via both 5-HT and SP}

Together, the above results indicate that spontaneous raphé neuron activity leads to endogenous release of both 5-HT and SP, and their concerted effects are critical for generation of the inspiratory rhythm and motor output both in the juvenile system in situ and in neonatal medullary slices. The next question is whether increased raphé neuronal activity (and increased release of these neuromodulators above basal levels) can increase network excitability both in vitro and in situ. Thus, we experimentally activated neurons in $\mathrm{n}$. raphé obscurus by local microinfusion of the glutamate receptor agonist AMPA in the ventral part of the nucleus (see box in Fig. 1C), analyzed the relation between raphé neuron firing and respiratory network output, and pharmacologically probed for release of endogenous transmitters induced by n. raphé obscurus stimulation.

\section{Raphé stimulation in vitro}

Inspiratory output was recorded from XII nerve rootlets in vitro simultaneously with extracellular potentials of midline raphé neurons (Fig. 10A). We controlled the level of raphé neuronal excitation and spiking frequency by the duration of the infusion and concentration of AMPA (1-5 $\mu \mathrm{M})$ in the pipette, which enabled us to cover a range of spiking rates that overlapped the physiological range of spiking observed for raphé neurons in vivo (Mason, 1997). For the neuron shown in Figure 10 A, microinfusion of AMPA $(5 \mu \mathrm{M})$ into the $\mathrm{n}$. raphé obscurus progressively increased firing rate from $1.2 \mathrm{~Hz}$ to a maximum of $7.8 \mathrm{~Hz}$ near the termination of infusion, while increasing inspiratory burst fre- 
quency by a factor of 1.9. On average, with our AMPA microinfusion protocols the firing rate of raphé neurons increased from $1.1 \pm 0.70$ to $14.24 \pm 0.25 \mathrm{~Hz}(p<$ $0.001 ; n=17)$ with $5 \mu \mathrm{M}$ AMPA, and increased the inspiratory frequency by a factor of $3.86 \pm 0.48(p<0.001 ; n=20$ slices). We found a monotonic relationship between the mean firing rate of raphé obscurus neurons, experimentally increased by local application of different AMPA concentrations, and inspiratory burst frequency (Fig. 10B). Based on Figure $2 C$, the maximum firing rate of raphé neurons that could be achieved with AMPA stimulation was equivalent to 50-100 pA of depolarizing current. We note that the effect of 5-HT neuron stimulation on inspiratory output may be blunted by the loss of some axonal projections from 5-HT neurons to the respiratory network due to the slicing procedure.

We repeated the local AMPA $(5 \mu \mathrm{M})$ microinfusion during bath application of 5-HT and SP antagonists. In this case, the increase in inspiratory frequency was partially antagonized by either MeSG ( 5 $\mu \mathrm{M})(42.8 \pm 2.6 \% ; p<0.001 ; n=17)$ (Fig. 10C, top) or KET $(7.5 \mu \mathrm{m})(32.0 \pm$ $3.7 \%$; $p<0.001 ; n=12$ ) (Fig. $10 D$, middle). Coapplying the NK-1 receptor antagonist SR $(7.5 \mu \mathrm{M})$ with MeSG $(5 \mu \mathrm{M})$ reduced the stimulatory effect of AMPA infusion in the raphé obscurus by $82.8 \pm$ 4.6\% $(p<0.001 ; n=17)$ (Fig. 10C, top). Similarly, coapplying SR $(7.5 \mu \mathrm{M})$ with KET reduced stimulation by $84.9 \pm$ 5.3\% ( $p<0.001 ; n=12)$ (Fig. 10C, middle). Coapplying MDL (10 $\mu \mathrm{M}, n=$ 5) with SR (Fig. 10C, bottom) also significantly attenuated activation of the respiratory network by raphé obscurus stimulation by $79 \pm 6.5 \%(p<0.001)$.

\section{Raphé stimulation in situ}

We performed similar raphé obscurus stimulation experiments in the in situ perfused preparations. Microinfusion pipettes were advanced from the dorsal surface of the medulla, at the rostrocaudal level of the preBötC, into the ventral part of raphé obscurus adjacent to the midline, where there is a high density of cells colocalizing 5-HT and SP (see Fig. 1C). We simultaneously recorded raphé neuronal population activity from a separate electrode attached to the microinfusion pipette assembly and positioned the pipettes at sites with tonic and typically rhythmic respiratory-related raphé neuronal activity (Fig. 11A), which was a signature of raphé obscurus. Recording/stimulation sites were also verified histologically in three preparations by small electrolytic lesions made by passing constant current (see Materials and Methods) through the tungsten recording elec-
D

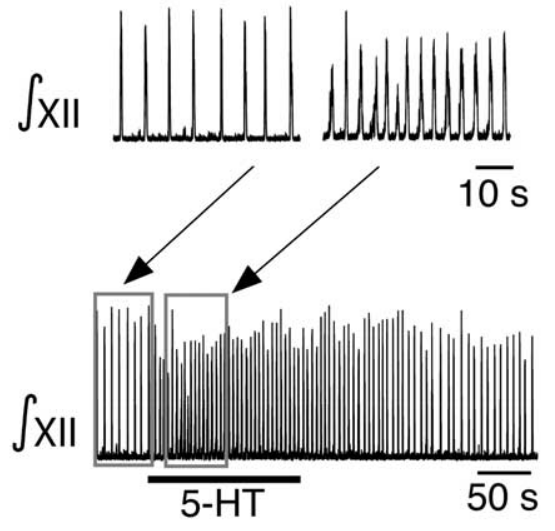

E
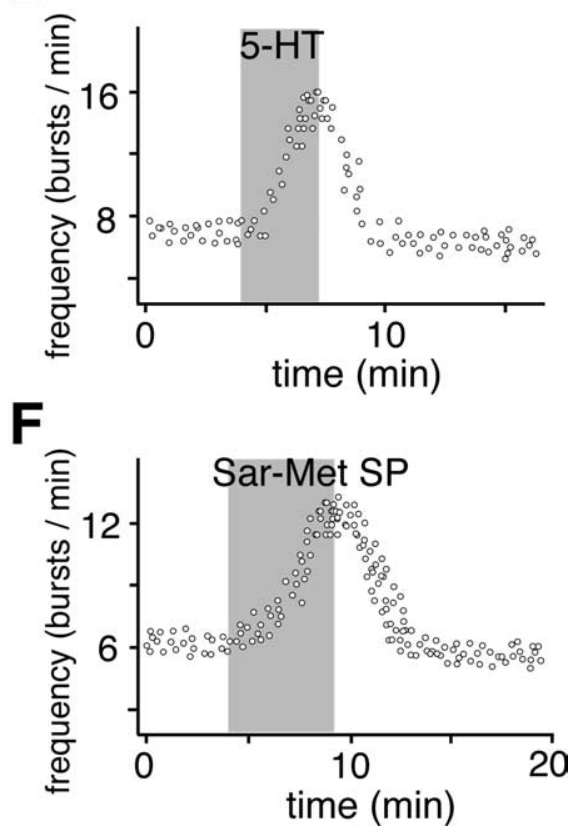

Figure 6. Spontaneous inspiratory activity depends on activation of 5-HT 2 and NK-1 receptors within the pre-BötC in vitro. $A-C$, Inspiratory frequency, as monitored by continuous recording of XII motoneuron population activity, is reversibly reduced receptor antagonist SR $140333(7.5 \mu \mathrm{M})(\mathbf{C})$. D, Recording of XII motor output ( $\left.\int \mathrm{XII}\right)$, showing the effects of bilateral microinfusion of 5-HT (30 $\mu \mathrm{m})$ into the pre-BötC, which increased inspiratory frequency and decreased burst amplitude. $\boldsymbol{E}, \boldsymbol{F}$, Inspiratory frequency plotted as function of time, showing reversible augmentation of inspiratory frequency by microinfusion of 5 - $\mathrm{HT}$ ( $30 \mu \mathrm{M})$ $(\boldsymbol{E})$ or Sar-Met SP $(1 \mu \mathrm{m})(\boldsymbol{F})$, a selective NK-1 receptor agonist, into the pre-BötC.

trode, confirming in each case that the AMPA microinfusion and recording sites were within the ventral aspect of raphé obscurus. As in slice preparations, microinfusion of AMPA (5 $\mu \mathrm{M})$ at these sites progressively increased local population activity and simultaneously augmented inspiratory discharge frequency recorded on phrenic and XII nerves (Fig. 11A,B). This excitation of inspiratory activity $(51.67 \pm 20.50 \%$ increase over control frequency, $n=4, p<0.001$ ) was also partially attenuated after application of SR, with or without MeSG, to the perfusate solution. Applying the NK-1 receptor antagonist SR $(20 \mu \mathrm{M})$ reduced the stimulatory effect of AMPA infusion in the raphé obscurus on frequency to $19.29 \pm$ 
A
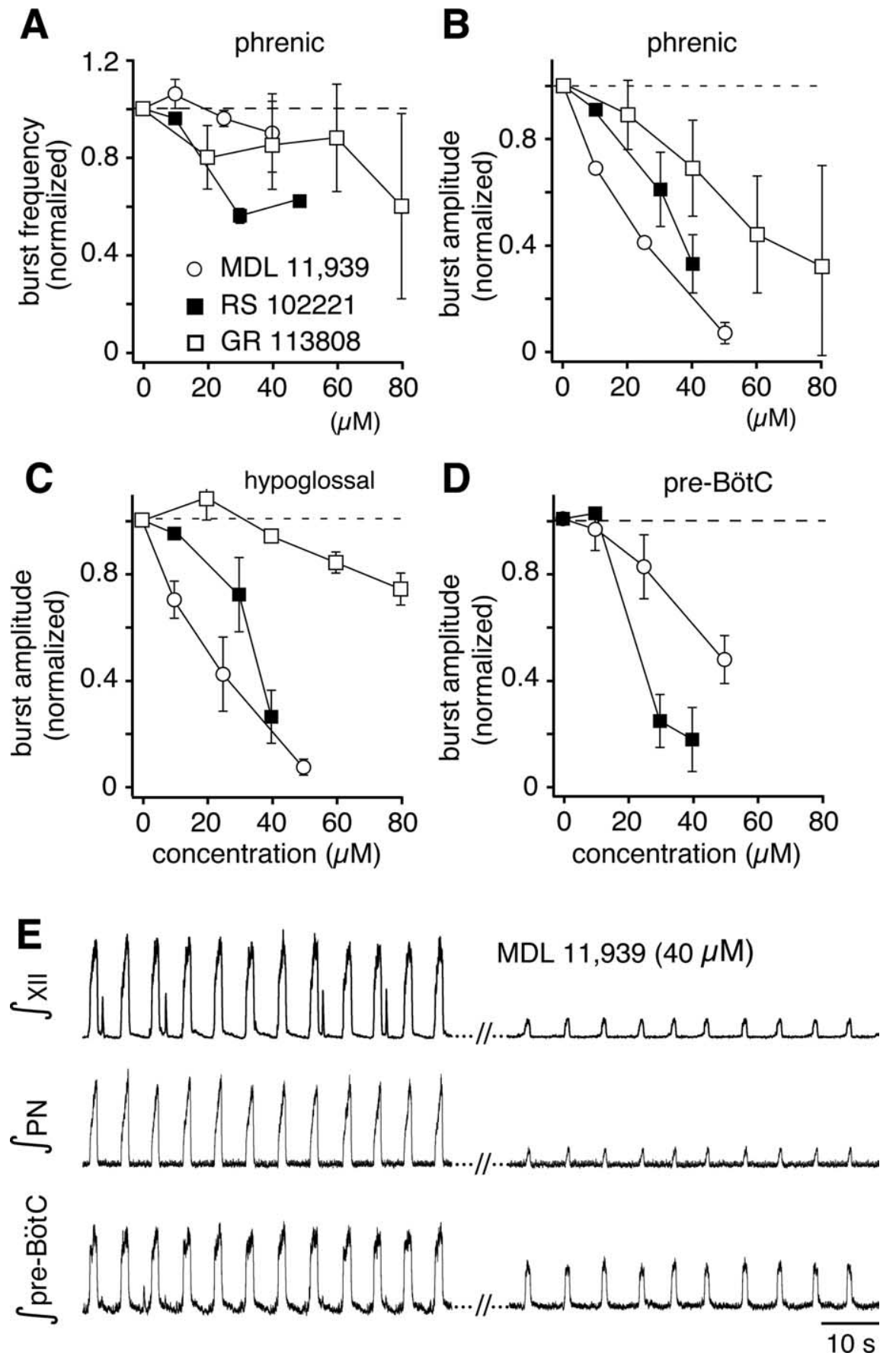

Figure 7. Endogenous 5-HT release is critical for inspiratory pattern generation and motor output in situ through multiple 5-HT receptor subtypes. $A$, Steady-state inspiratory burst frequency versus concentration of $5-\mathrm{HT}_{2 \mathrm{~A}}(\mathrm{MDL} 11,939), 5-\mathrm{HT}_{2 \mathrm{C}}$ (RS 102221), and $5-\mathrm{HT}_{4}(\mathrm{GR} 113808)$ receptor antagonists applied via the perfusate in situ. Only the $5-\mathrm{HT}_{2 \mathrm{C}}$ antagonist significantly decreased burst frequency (shown normalized to control values, data points are mean values \pm SEM) indicating a $5-\mathrm{HT}_{2 \mathrm{C}}$ receptor component for modulation of inspiratory rhythm generation. $\boldsymbol{B}-\boldsymbol{D}$, All three antagonists decreased the amplitudes (normalized to control, mean values \pm SEM) of integrated inspiratory bursts in motor nerves (PN in $\boldsymbol{B}, \mathrm{XII}$ in $\boldsymbol{C}$ ) and pre-BötC (D) population activity in a concentration-dependent manner, indicating involvement of multiple receptor subtypes in regulation of inspiratory activity amplitude. The larger antagonism of PN activity compared with XII output by GR 113808 suggests differential contributions of $5-\mathrm{HT}_{4}$ receptors in regulation of spinal and cranial motor outputs. $\boldsymbol{E}$, Representative recordings of integrated XII, $\mathrm{PN}$, and pre-BötC population activity recorded in the in situ preparation illustrating typical large reductions of inspiratory burst amplitudes with only small perturbation of burst frequency by antagonism of $5-\mathrm{HT}_{2 \mathrm{~A}}$ receptors with MDL 11,939.

$19.63 \%$ of control $(n=4, p<0.001)$. Coapplying MeSG $(20$ $\mu \mathrm{M})$ with SR further reduced the stimulatory effect of AMPA infusion in the raphé obscurus to $8.52 \pm 6.79 \%$ of control $(n=$ $4, p<0.05$ ) (Fig. 11C,D).
Raphé neurons excite XII motoneurons and pre-BötC inspiratory neurons in vitro via 5 -HT and SP

Excitation of XII motoneurons

Stimulation of raphé neurons by local AMPA microinfusion also caused increased tonic activity in XII nerve motor output (35/39 slices) (Fig. 10A). The increase in tonic activity was reduced (29/35 slices) by bath-applied MeSG $(5 \mu \mathrm{M})$, and further reduced by bath coapplication of MeSG and SR (7.5 $\mu \mathrm{M})$ (data not shown). To determine whether this increase in tonic activity resulted from depolarization of XII motoneurons, we performed wholecell recordings from identified inspiratory XII motoneurons; these cells depolarized (4.9 $\pm 1.2 \mathrm{mV} ; n=12$ neurons) during local AMPA microinfusion in the n. raphé obscurus. This tonic excitation was not observed during local microinfusion of 5 -HT or SP into the pre-BötC (Fig. 6D), suggesting that it was caused by the release of 5-HT and SP directly into the hypoglossal nuclei. To test this hypothesis, we blocked glutamatergic synaptic transmission with CNQX $(20 \mu \mathrm{M})$ in the bath solution to eliminate rhythmic inspiratory synaptic drive (Funk et al., 1993), and we stimulated raphé neurons with local microinfusion of elevated $\left[\mathrm{K}^{+}\right](15 \mathrm{mM})$ into the $\mathrm{n}$. raphé obscurus. This induced a depolarization of XII motoneurons (4.4 \pm $1.4 \mathrm{mV} ; n=12$ ) (Fig. 12A) similar to that when AMPA was applied. Bath application of MeSG $(5 \mu \mathrm{M})$ significantly reduced this depolarization to $2.3 \pm 0.6 \mathrm{mV}(p<$ $0.001 ; n=7)$, while coapplying SR further attenuated it to $1.4 \pm 0.4 \mathrm{mV}(p<0.001$; $n=7)$. Similarly, bath-applied KET (7.5 $\mu \mathrm{M})$ significantly reduced the depolarization to $3.0 \pm 1.3 \mathrm{mV}(p<0.001 ; n=6)$ (Fig. 12A), while coapplying SR 140333 further attenuated the depolarization to $1.9 \pm 0.8 \mathrm{mV}(p<0.001 ; n=7)$ (Fig. $12 \mathrm{~A})$. Thus, these results indicate that raphé obscurus neurons directly excite XII motoneurons by releasing 5-HT and SP. This finding is consistent with raphé neuron axonal projections to the XII motor nucleus (Fig. 3E) and colocalization of 5-HT and SP in a subset of synaptic terminals in this region (supplemental Fig. 1, available at www.jneurosci.org as supplemental material).

\section{Excitation of pre-BötC neurons}

There are two main electrophysiological phenotypes of inspiratory neuron in the pre-BötC (Koshiya and Smith, 1999; Del Negro et al., 2002; Peña and Ramirez, 2004; Peña et al., 2004): intrinsically bursting neurons that continue to generate rhythmic bursts after excitatory synaptic transmission is blocked, and non- 
intrinsic bursters that become inactive in the absence of synaptic transmission (Koshiya and Smith, 1999). We studied both cell types, since both have been proposed to be involved in rhythm generation by excitatory synaptic interactions within the heterogeneous preBötC network (Butera et al., 1999a,b; Del Negro et al., 2001; Peña and Ramirez, 2004; Purvis et al., 2007).

We microinfused aCSF with elevated $\left[\mathrm{K}^{+}\right]$(15 mM) into the $\mathrm{n}$. raphé obscurus, to stimulate raphé neurons as described above, while recording from pre-BötC cells before or after rhythmic excitatory synaptic drive was blocked with bathapplied CNQX (20 $\mu \mathrm{M})$ (Koshiya and Smith, 1999). In all cases, we observed progressive depolarization during stimulation of raphé obscurus neurons. In CNQX, intrinsic bursters $(n=14)$ depolarized by $12.5 \pm 3.7 \mathrm{mV}$; nonintrinsic bursters $(n=5)$ were similarly depolarized (by $10.2 \pm 3.1 \mathrm{mV}$ ). In CNQX, all intrinsic bursters exhibited increased burst frequency during this depolarization (Fig. 12B), consistent with the voltage-dependent bursting behavior described for these cells (Butera et al., 1999a; Del Negro et al., 2002). Four of these intrinsic bursters depolarized into tonic spiking after transitioning from voltagedependent bursting (Fig. 12B1). Bath application of MeSG (5 $\mu \mathrm{M} ; n=3$; data not shown) or $\operatorname{KET}(7.5 \mu \mathrm{M} ; n=4)$ (Fig. 12B2) reduced the baseline intrinsic burst frequency and attenuated the membrane depolarization induced by raphé stimulation. Coapplying SR further reduced the stimulus-induced membrane depolarization $(n=4)$ (Fig. $12 \mathrm{B3})$. A similar attenuation of the depolarization by KET and SR was observed in nonintrinsic bursters in CNQX ( $n=5$; data not shown). These results confirm that 5-HT and SP are both released in the pre-BötC during raphé stimulation and contribute to neuronal depolarization via $5-\mathrm{HT}_{2 \mathrm{~A} / 2 \mathrm{C}}$ and $\mathrm{NK}-1$ receptor activation.

\section{Serotonin modulates pre-BötC neurons and XII motoneurons via cation leak currents}

Pre-BötC neurons

To analyze biophysical mechanisms underlying depolarization of pre-BötC neurons induced by raphé stimulation, we investigated in greater detail the effects of bath-applied 5-HT on membrane potential and bursting behavior of pre-BötC neurons. Thus, 5-HT $(30 \mu \mathrm{M})$ significantly depolarized intrinsically bursting neurons (Fig. 13A) from $-65.8 \pm 8.1 \mathrm{mV}$ to $-53.4 \pm 8.0 \mathrm{mV}$ $(p<0.01 ; n=5)$. Similarly, nonintrinsically bursting neurons were depolarized by 5 -HT from $-61.4 \pm 6.1 \mathrm{mV}$ to $-52.8 \pm 5.4$ $\mathrm{mV} ; p<0.01 ; n=5)$. In voltage clamp at a holding potential of $-70 \mathrm{mV}, 5-\mathrm{HT}$ increased the net inward current in intrinsically bursting neurons from $-50.3 \pm 6.8$ to $-65.6 \pm 6.7 \mathrm{pA}(p<0.01$; $n=6)$ and in nonintrinsically bursting neurons from $-52.8 \pm$ $8.1 \mathrm{pA}$ to $-71.4 \pm 7.9 \mathrm{pA}(p<0.001 ; n=5)$.

These results suggest that excitatory actions of $5-\mathrm{HT}$ in preBötC cells involve cation conductances that are active at subthreshold membrane voltages, such as currents contributing to total background (leak) currents, as previously hypothesized for pre-BötC inspiratory neurons (Schwarzacher et al., 2002) and
B

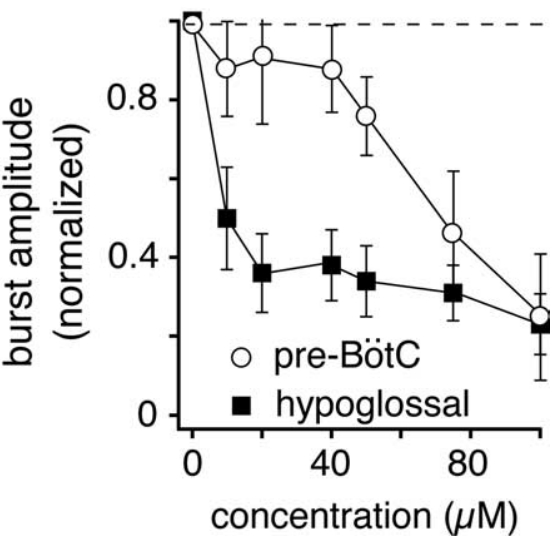

Figure 8. NK-1 receptor activation in situ contributes to maintenance of inspiratory burst amplitude. $\boldsymbol{A}$, Blocking endogenous

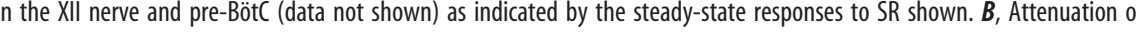

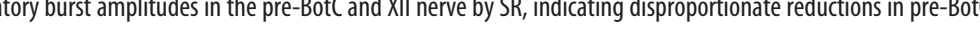
inspiratory burst generation downstream from the pre-BötC. Data points represent mean values \pm SEM.

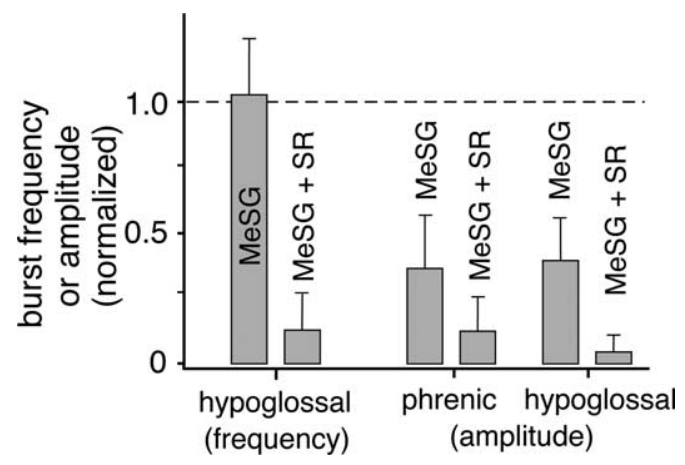

Figure 9. Serotonin and substance $P$ in concert are required to generate and maintain the respiratory rhythm and motor output in situ. Antagonism of $5-\mathrm{HT}_{1,2}$ receptors with the nonselective receptor antagonist MeSG $(20 \mu \mathrm{m})$ did not affect inspiratory rhythm generation, as shown by the lack of effect on inspiratory burst frequency (XII/PN), but strongly reduced PN and XII nerve burst amplitudes. Addition of the NK-1 antagonist SR (20 $\mu \mathrm{m})$ nearly eliminated all inspiratory burst activity, as shown by the significant large decreases in burst frequency and amplitudes. This suggests that endogenous 5-HT and NK-1 receptor activation work in concert to generate and maintain inspiratory activity in situ. Error bars indicate SEM.

demonstrated for other neurons (Hwang and Dun, 1999; Talley et al., 2000). Thus, we compared changes in the $I-V$ relations in both intrinsically bursting and nonintrinsically bursting neurons under voltage-clamp conditions during slow voltage ramps (30 $\mathrm{mV} / \mathrm{s},-90$ to $0 \mathrm{mV}$ ) before and after bath application of 5-HT (Fig. 13B-D).

Without channel blockers and using regular internal solution, 5 -HT caused a rightward shift of the ramp $I-V$ relation, corresponding to a change in the total leak equilibrium potential $\left(E_{\text {Leak }}\right)$, from initial values of $-60.3 \pm 4.7 \mathrm{mV}$ and $-59.7 \pm 4.3$ $\mathrm{mV}$ for intrinsically bursting $(n=5)$ and nonintrinsically bursting inspiratory neurons $(n=5)$, respectively. This was associated with an increase of the average leak conductance of $71 \%$ (from $2.57 \pm 0.77$ to $4.4 \pm 0.9 \mathrm{nS}, n=7$, pooled values from four intrinsic bursters and three nonintrinsic bursters), as estimated from linear regression of $I-V$ relations over the voltage range -90 to $-70 \mathrm{mV}$ where the $I-V$ curve was linear (Fig. 13B1). The 5 -HT-induced current (Fig. 13B2), obtained by subtracting the 
A
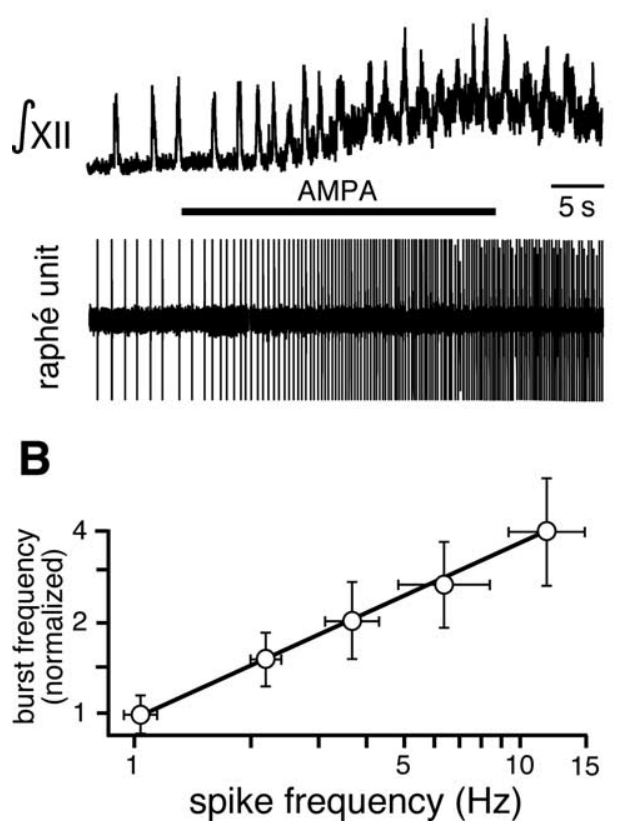

C

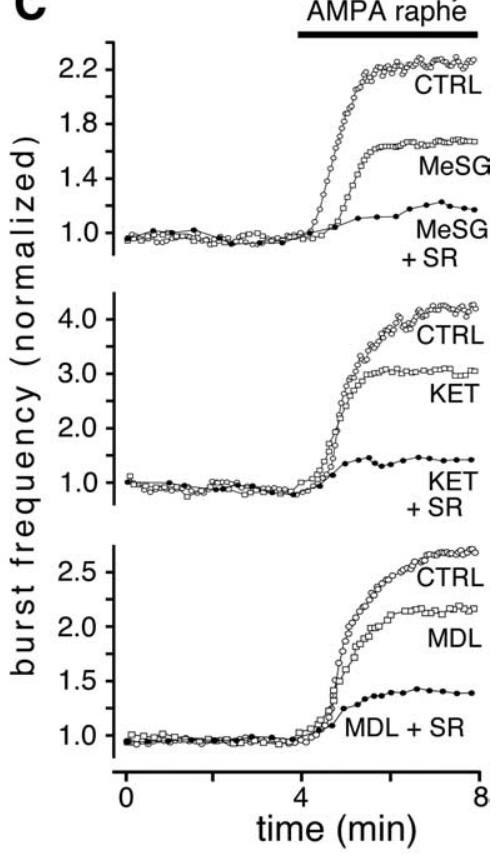

Figure 10. Increased firing of raphé neurons in slices stimulates inspiratory output via activation of $5-\mathrm{HT}_{2}$ and NK-1 receptors. A, XII motor output (top, $\int \mathrm{XII}$ ) recorded simultaneously with extracellular potential of a raphé obscurus neuron (bottom trace). AMPA $(5 \mu \mathrm{m})$ microinfused into the $\mathrm{n}$. raphé obscurus progressively increased firing rate of the raphé neuron (6.5-fold) and inspiratory frequency (1.9-fold). There was also an increase in tonic activity in hypoglossal motoneurons, as seen by the shift in baseline of the integrated XII population activity. $\boldsymbol{B}$, Relationship between inspiratory burst frequency (normalized to baseline) and average firing rate of raphé neurons ( $n=12$ slices). Data points are mean values \pm SEM. C, Top, The excitatory effect of raphé stimulation on burst frequency [control (CTRL)] was partially blocked by bath-applied MeSG $(5 \mu \mathrm{M})$, and nearly eliminated by coapplication of SR 140333 (MeSG + SR;7.5 $\mu$ M). Middle, The excitatory effect was also partially blocked by bath-applied KET (7.5 $\mu \mathrm{M})$, and further attenuated by coapplying SR 140333 (KET + SR). Bottom, MDL 11,939 (10 $\mu \mathrm{M})$ also partially blocked the excitation, which was also further attenuated by coapplying $7.5 \mu \mathrm{M} S R(M D L+S R)$.

control $I-V$ curve from that obtained in the presence of $5-\mathrm{HT}$, was nearly linear from -90 to $-30 \mathrm{mV}$ with an average reversal potential of $-40.8 \pm 3.4 \mathrm{mV}$. After blocking $\mathrm{Na}^{+}$channels with TTX $(1 \mu \mathrm{M})$, we still observed a depolarizing shift of $I-V$ curves (Fig. 13C1) and increase of conductance (65\%: from $2.24 \pm 0.63$ to $3.65 \pm 0.86 \mathrm{nS}, p<0.01)$; the difference current was similar to that without TTX (Fig. 13C2) with an average reversal potential of $-39.1 \pm 2.9 \mathrm{mV}(n=4)$, indicating that TTX-sensitive $\mathrm{Na}^{+}$ channels are not involved. An essentially identical difference current was obtained when we simultaneously blocked voltageactivated $\mathrm{Na}^{+}$and $\mathrm{K}^{+}$currents, respectively, with TTX and TEA (20 mM; $n=4$, data not shown). We also tested whether the hyperpolarization-activated nonselective cation current $\left(I_{\mathrm{h}}\right)$ was involved, since 5-HT has been shown to augment this current in some neurons (Bobker and Williams, 1989; Gasparini and DiFrancesco, 1999). The depolarizing shift of $I-V$ curves and the difference current induced by 5 -HT were not affected by the $I_{\mathrm{h}}$ blocker ZD 7288 (100 $\mu \mathrm{M} ; n=6$; data not shown).

In some neurons, several background cation currents are simultaneously modulated by 5-HT (Hwang and Dun, 1999). We evaluated the contribution of leak cation conductances in a group of pre-BötC inspiratory neurons $(n=7)$ by blocking voltagegated $\mathrm{Na}^{+}, \mathrm{K}^{+}$, and $\mathrm{Ca}^{2+}$ channels as well as background $\mathrm{K}^{+}$ currents with $1 \mu \mathrm{M}$ TTX, $20 \mathrm{~mm}$ TEA-Cl, and $200 \mu \mathrm{M} \mathrm{CdCl}_{2}$ and using a $\mathrm{Cs}^{+}$-based internal solution. This essentially linearized the total $I-V$ curve (Fig. 13D1). Under these conditions 5-HT still induced an increase in the average membrane conductance (Fig. 13D1) (by 29\%: from $1.9 \pm 0.57$ to $2.41 \pm 0.62 \mathrm{nS}, p<0.01$,

pooled values from $n=2$ pre-BötC intrinsic bursters and $n=5$ nonintrinsic bursters); the 5-HT-induced current (Fig. $13 D 2)$, obtained by $I-V$ curve subtraction, was still linear, but it was smaller and the average reversal potential was shifted to more depolarized values $(-7.5 \pm 5 \mathrm{mV}$; $n=7)$ than without internal $\mathrm{Cs}^{+}$. A potential explanation for these results is that a background $\mathrm{K}^{+}$conductance(s) is affected at the same time that a TTXinsensitive, nonselective cation conductance is increased in pre-BötC neurons by 5-HT.

We also analyzed conductance mechanisms underlying neuronal depolarization by SP. In the cells studied $(n=10)$, SP (1-2 $\mu \mathrm{M}$ ) shifted the total $I-V$ relation and $E_{\text {Leak }}$ in a manner similar to that of 5-HT. The leak current induced under conditions in which voltage-activated channels were blocked had $I-V$ characteristics and a reversal potential similar to that induced by 5-HT (supplemental Fig. 2, available at www.jneurosci.org as supplemental material). Hayes and Del Negro (2007) recently found a similar SP-induced nonselective cation current in neonatal mouse preBötC neurons, and our new results with 5-HT suggest that both neuromodulators share a similar mechanism of excitation of pre-BötC cells via cation conductances contributing to the total leak current.

Previous studies have shown that a voltage-dependent persistent sodium current $\left(I_{\mathrm{NaP}}\right)$ is augmented in pre-BötC neurons of neonatal mice by activation of $5-\mathrm{HT}_{2 \mathrm{~A}}$ receptors (Peña and Ramirez, 2002). $I_{\mathrm{NaP}}$ is common in the two types of pre-BötC neurons (Del Negro et al., 2002; Koizumi and Smith, 2008) analyzed here. We did not find a significant effect of 5-HT on this current (supplemental Fig. 3, available at www.jneurosci.org as supplemental material) $(n=7)$ in neurons in which leak currents were altered as described above.

\section{XII inspiratory motoneurons}

We analyzed conductance mechanisms underlying the depolarization of XII motoneurons by 5-HT and SP. We evaluated the contribution of leak cation conductances in a group of inspiratory motoneurons $(n=6)$ by blocking voltage-gated $\mathrm{Ca}^{2+}$ channels with $200 \mu \mathrm{M} \mathrm{CdCl}_{2}$ and recording $I-V$ relations obtained with $30 \mathrm{mV} / \mathrm{s}$ voltage-clamp ramps as described above. 5-HT (10 $\mu \mathrm{M})$ consistently produced a shift of the $I-V$ relation similar to that in pre-BötC neurons (Fig. 13B1) and increased the total leak conductance (from $13.9 \pm 3.1$ to $17.0 \pm 3.1 \mathrm{nS}, n=3$ ). The reversal potential for the induced current was $-18.7 \pm 6.5 \mathrm{mV}$, obtained from the 0 current intercept of the difference (before and after 5-HT) $I-V$ relationship. In contrast, $S P$ decreased the total leak conductance (from $13.1 \pm 4.0$ to $9.3 \pm 3.1$ ), and the reversal potential of the induced current was $-70.8 \pm 8.1 \mathrm{mV}$, which is close to the $\mathrm{K}^{+}$equilibrium potential $(-72 \mathrm{mV})$ calculated for our recording conditions, suggesting that SP predominantly blocked a $\mathrm{K}^{+}$-dominated background conductance in the motoneurons. 
Serotonin converts a subset of nonintrinsically bursting pre-BötC neurons into intrinsic bursters We found that a subset of nonintrinsically bursting pre-BötC neurons $(n=4 / 10$ tested) was converted to intrinsic bursters by 5 -HT. After blocking synaptic transmission with bath-applied $\mathrm{CdCl}_{2}(200$ $\mu \mathrm{M})$, bath application of 5-HT $(30 \mu \mathrm{M})$ reversibly transformed the nonintrinsically bursting phenotype of these neurons into rhythmic bursting (Fig. 14). This bursting behavior was voltage dependent (Fig. $14 C$ ), similar to that normally manifested by intrinsically bursting pre-BötC neurons. Interestingly, these intrinsic bursting properties emerged more slowly than the time to peak depolarization of the neurons (average of $48.5 \mathrm{~s}$ to bursting vs $16.3 \mathrm{~s}$ to peak depolarization; $n=4$ ). We also tested SP. In all pre-BötC nonintrinsically bursting inspiratory neurons examined $(n=$ 10), SP depolarized the cells but did not convert them to intrinsic bursting behavior. Thus, 5-HT not only depolarizes preBötC neurons to increase network bursting frequency, but can also transform the electrophysiological phenotype of some pre-BötC neurons to bursters. There is controversy regarding whether bursting properties are necessary for generation of the respiratory rhythm (Del Negro et al., 2005; Pace et al., 2007; Koizumi and Smith, 2008), but there is no doubt that development of intrinsic bursting could enhance intrinsic rhythmicity of the preBötC network (Purvis et al., 2007).

\section{Discussion}

Raphé obscurus neurons project to and excite neurons in the pre-BötC and XII motor nucleus

The mechanisms by which raphé 5-HT neurons modulate respiratory circuit function have not been clearly established (Richter et al., 2003; Richerson, 2004). Although 5-HT can have either inhibitory or excitatory actions on various respiratory neurons (Richter et al., 2003), there is controversy regarding whether the overall effect of raphé neurons is to stimulate or inhibit respiratory motor output in vivo (Olson et al., 1979; Lalley, 1986; Richter et al., 2003; Richerson, 2004), whether raphé modulation is essential for respiratory motor output generation (Peña and Ramirez, 2002; Toppin et al., 2007; St.-John and Leiter, 2008), and which subpopulations of 5-HT neurons are involved. Our novel results establish that both 5-HT and SP are released endogenously from the raphé system and are critical for maintenance of respiratory motor output, both in the neonatal system in vitro and the juvenile system in situ, and this is due to excitation at the level of the pre-BötC and motoneurons. We focused on a particular subpopulation of 5-HT neurons, in the n. raphé obscurus, which we demonstrated is spontaneously active, has reciprocal connections with the respiratory network, and excites pre-BötC neurons and XII motoneurons to augment inspiratory activity.
B
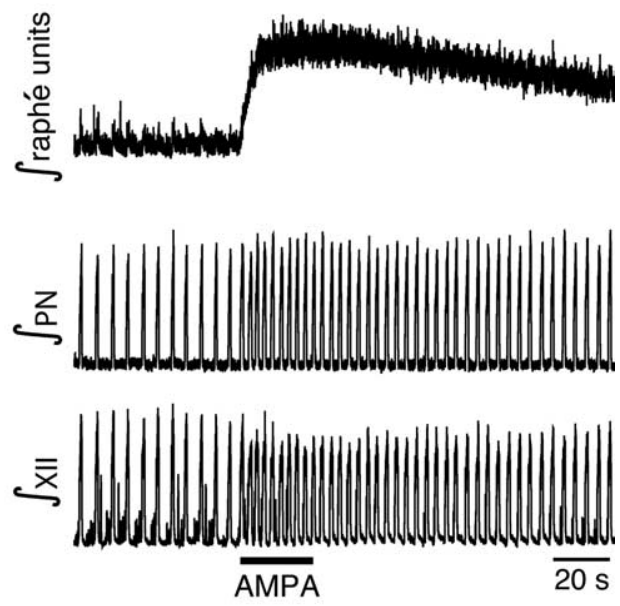

D

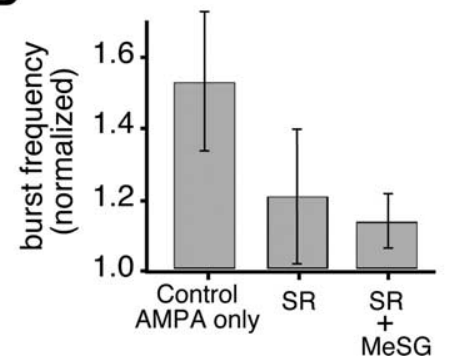

Figure 11. The raphé obscurus in situ drives frequency modulation of the respiratory network through 5-HT and NK-1 receptors. $\boldsymbol{A}$, Simultaneous recordings of population activity in the raphé obscurus (raw and integrated multiunit activity shown) and

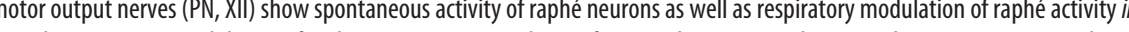
ins MeSG, showing strong attenuation of peak responses by the antagonists. These data indicate functional connectivity between the raphé obscurus and inspiratory rhythm-generating components of the respiratory circuit mediated by 5-HT and NK-1 receptors.

This excitation also results from release of both 5-HT and SP, which modulate cation leak conductances. Our use of multiple selective antagonists provides strong evidence that the modulatory effects were primarily due to $5-\mathrm{HT}_{2 \mathrm{~A}}$ and $\mathrm{NK}-1$ receptor activation, probably with a coexisting role of $5-\mathrm{HT}_{2 \mathrm{C}}$ and $5-\mathrm{HT}_{4}$ receptors.

Other 5-HT receptor subtypes expressed in the respiratory network have also been implicated in the control of breathing. For example, $5-\mathrm{HT}_{1 \mathrm{~A}}$ receptors are extensively expressed (Richter et al., 2003), and 5- $\mathrm{HT}_{1 \mathrm{~A}}$ agonists inhibit some respiratory neurons (Lalley et al., 1994), including some pre-BötC cells (Schwarzacher et al., 2002). As proposed by Richter et al. (2003), specific inhibitory neurons that contribute to inspiratory-expiratory phase switching in the intact respiratory network in vivo may be particularly sensitive to inhibition by $5-\mathrm{HT}_{1 \mathrm{~A}}$ receptor activation, which could disinhibit the network. We found a small residual excitation during augmented raphé neuron activity following block of $5-\mathrm{HT}_{2 \mathrm{~A}}$ and NK-1 receptors (Fig. 10C), which may reflect activation of 5- $\mathrm{HT}_{1 \mathrm{~A}}, 5-\mathrm{HT}_{4}$ (Manzke et al., 2003; Richter et al., 2003), 5- $\mathrm{HT}_{7}$, or non-5-HT receptors. In particular, we have not investigated the role of thyrotropin-releasing hormone (TRH), which is colocalized 


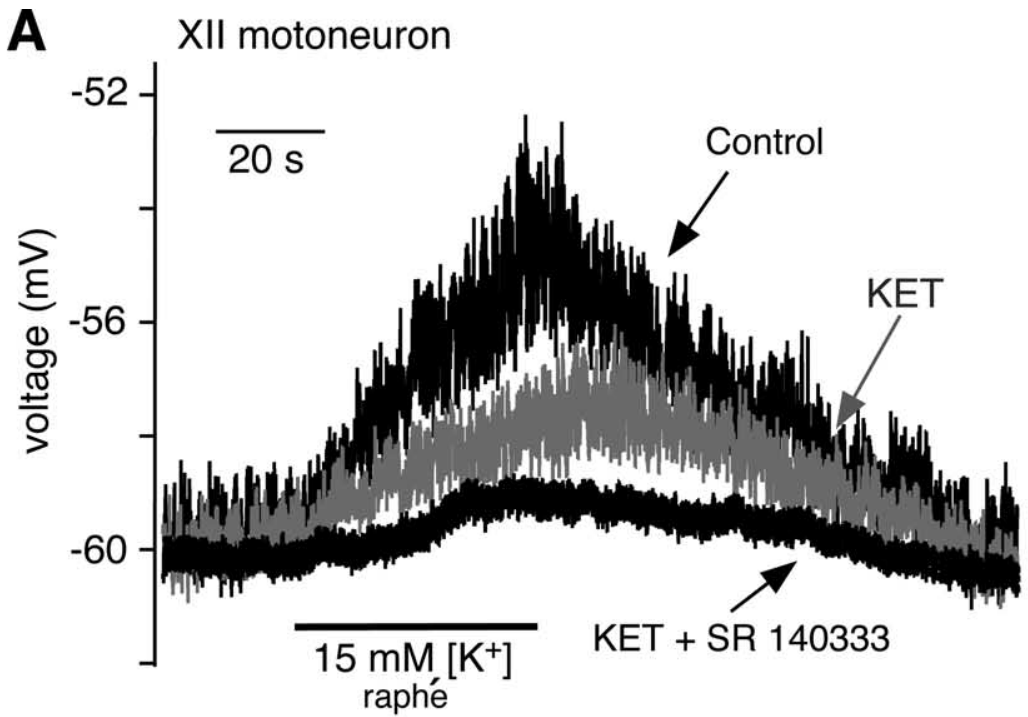

B1 pre-BötC neuron
Control

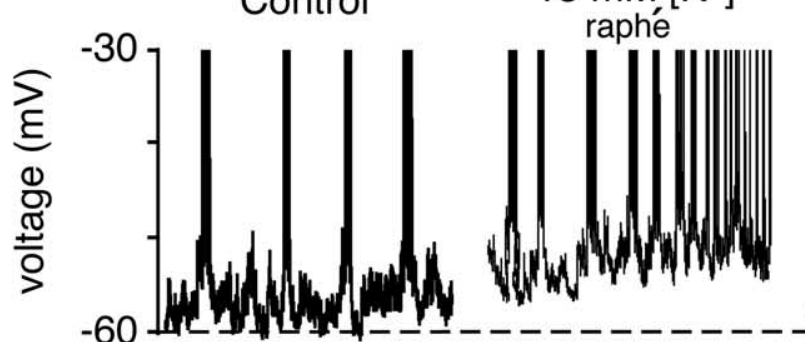

B2 $-60$

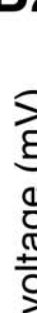

KET

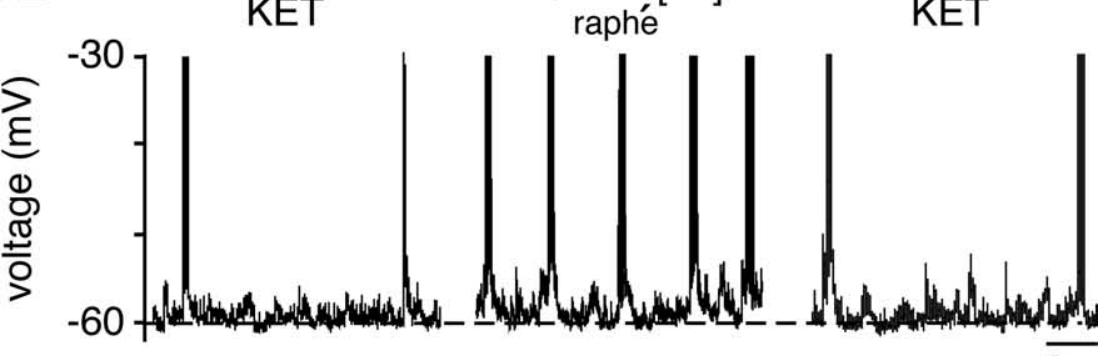

B3

$\mathrm{KET}+\mathrm{SR}$

$15 \underset{\text { raphé }}{15 \mathrm{~m}}\left[\mathrm{~K}^{+}\right]$

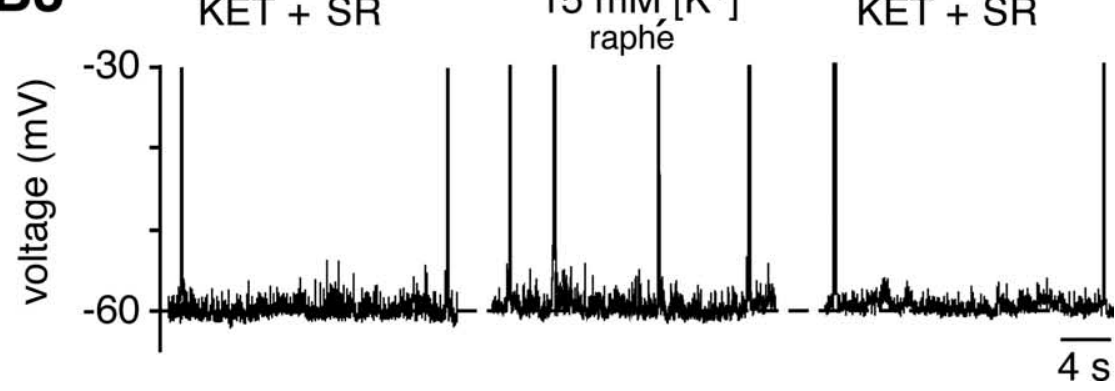

Figure 12. Serotonin and SP released from n. raphéobscurus depolarize inspiratory XII motoneurons and pre-BötCneurons in slices. $A$, Whole-cell current-clamp recording of XII motoneuron that depolarized in response to focal microinfusion of aCSF with elevated $\mathrm{K}^{+}(15$ $\mathrm{mm}$ ) into the $\mathrm{n}$. raphé obscurus to stimulate serotonin neurons. CNQX (20 $\mu \mathrm{M})$ was present in the bath. This depolarization was partially blocked by bath-applied ketanserin $(7.5 \mu \mathrm{M})$ and further attenuated by coapplying SR $140333(7.5 \mu \mathrm{m}) . \mathbf{B 1}$, Example of intrinsically bursting pre-BötC neuron that was depolarized by raphé stimulation. This first augmented the intrinsic bursting frequency and then reversibly transformed the firing pattern from bursting to tonic spiking. $\boldsymbol{B}$, In the same neuron as $\boldsymbol{B}$, bath-applied KET (7.5 $\mu \mathrm{m})$ decreased the initial burst frequency (left) and attenuated the effect of raphé stimulation (middle). $B 3$, In the same neuron, coapplication of SR $14033(7.5 \mu \mathrm{m})$ further reduced the initial bursting frequency relative to KET alone (left, note the change in time scale relative to B2) and nearly abolished the excitation that occurred during raphé stimulation (middle). in some raphé neurons and is well known to have strong excitatory effects on respiratory output in vivo and in vitro (Richerson, 2004), because there is not a specific antagonist available.

There were differences in the modulatory actions of endogenous 5-HT between the isolated neonatal in vitro system and the more intact, mature system in situ. For example, inspiratory rhythm generation in situ was typically not disrupted under basal conditions by blocking $5-\mathrm{HT}_{2}$ or NK-1 receptors alone as in the neonatal system in vitro, but rhythm generation was disrupted by 5 -HT and SP acting in concert. This likely reflects developmental differences and/or preparation state-dependent differences, in which 5-HT alone may not play as dominant a role in a more mature and/or intact system.

\section{Raphé 5-HT neurons excite inspiratory} neurons via leak conductances

Serotonin affects multiple ionic currents in various neurons, including XII motoneurons (Bayliss et al., 1995, 1997; Talley et al., 2000; Carr et al., 2002; Xiang et al., 2005). Our data show that endogenously released 5-HT and SP depolarize pre-BötC neurons and XII motoneurons, which involves modulation of leak currents. Exogenous 5-HT, SP, and TRH have each been shown to depolarize XII motoneurons by inhibiting TASK channels (Talley et al., 2000). We have confirmed that SP primarily reduces a leak $\mathrm{K}^{+}$conductance in XII inspiratory motoneurons; 5-HT, however, operates simultaneously on other leak conductances, similar to actions of 5-HT on pre-BötC neurons.

Indeed our data indicate that $5-\mathrm{HT}_{2 \mathrm{~A}}$ receptor activation depolarizes pre-BötC inspiratory neurons by effects on the total leak current consistent with a simultaneous decrease in a $\mathrm{K}^{+}$leak conductance(s) and increase in a nonselective cation leak current, similar to findings for other medullary neurons (Hwang and Dun, 1999). We currently do not know the molecular identity of the $\mathrm{K}^{+}$and nonselective cation channels involved. TASK channels are candidates for the $\mathrm{K}^{+}$selective component of the leak current as in XII motoneurons (Talley et al., 2001). A reduction in TASK conductance (Talley et al., 2001) simultaneously with activation of the nonselective cation current could explain the depolarized shift of $E_{\text {Leak }}$ and associated neuronal depolarization. The recently described nonselective leak sodium current NALCN (Lu et al., 2007) has an $I-V$ relation and reversal potential very similar to that of the mixed-cationic cur- 
A
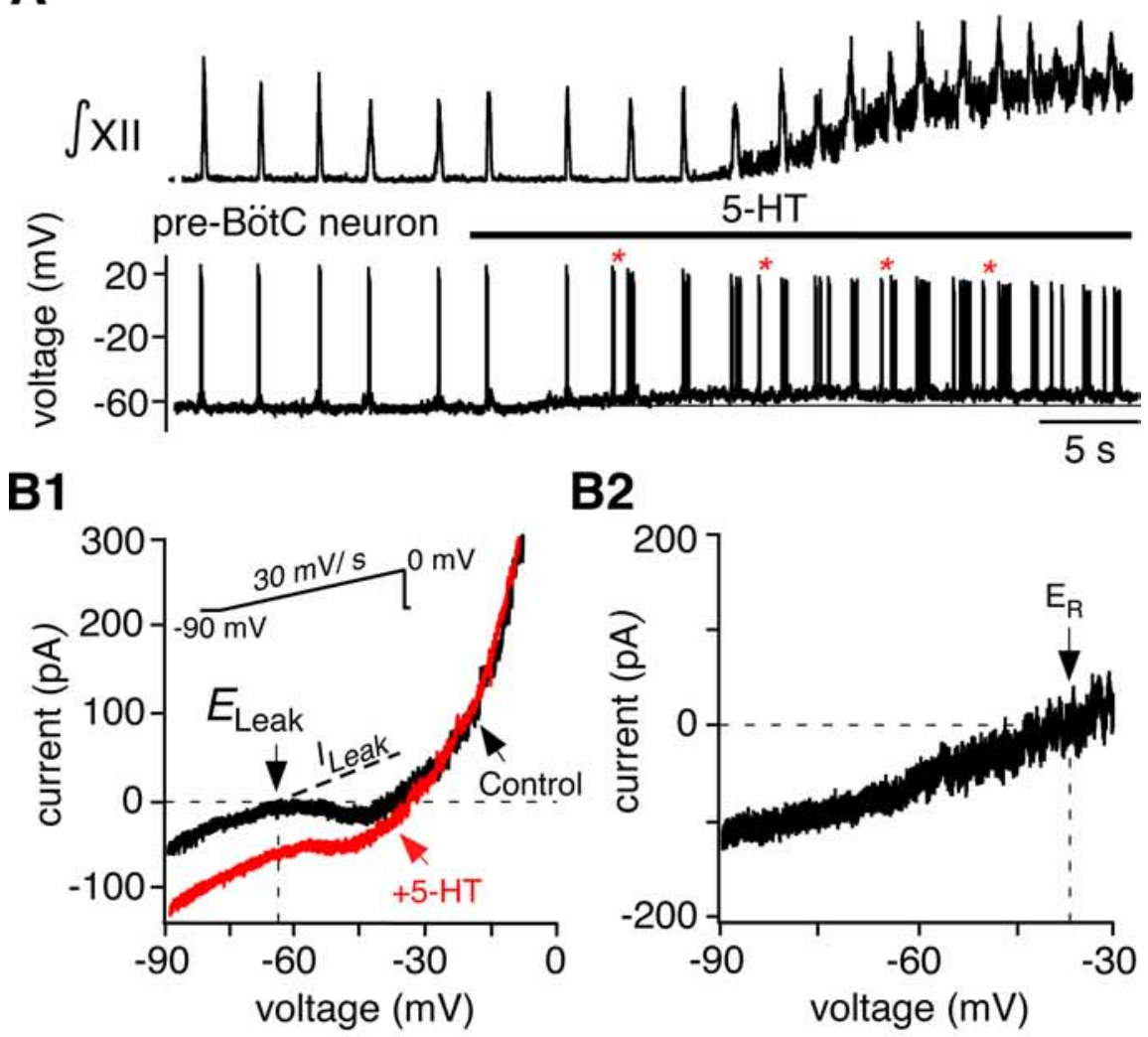

B2

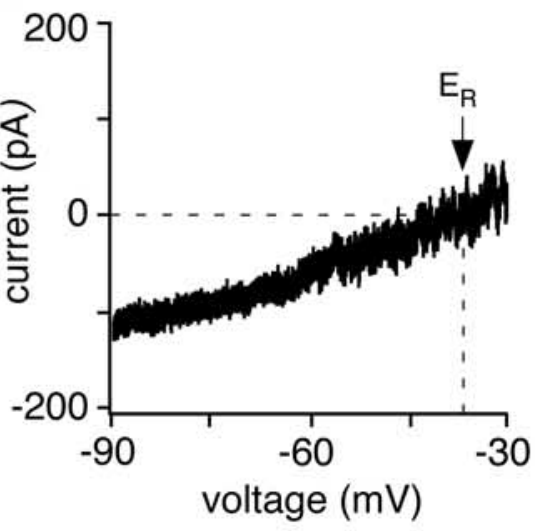

C1

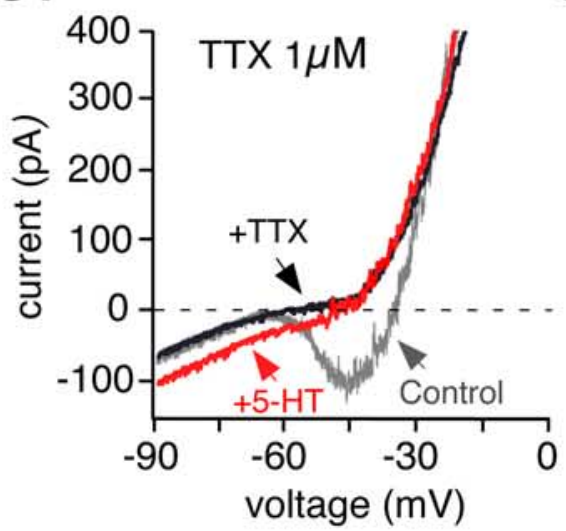

C2
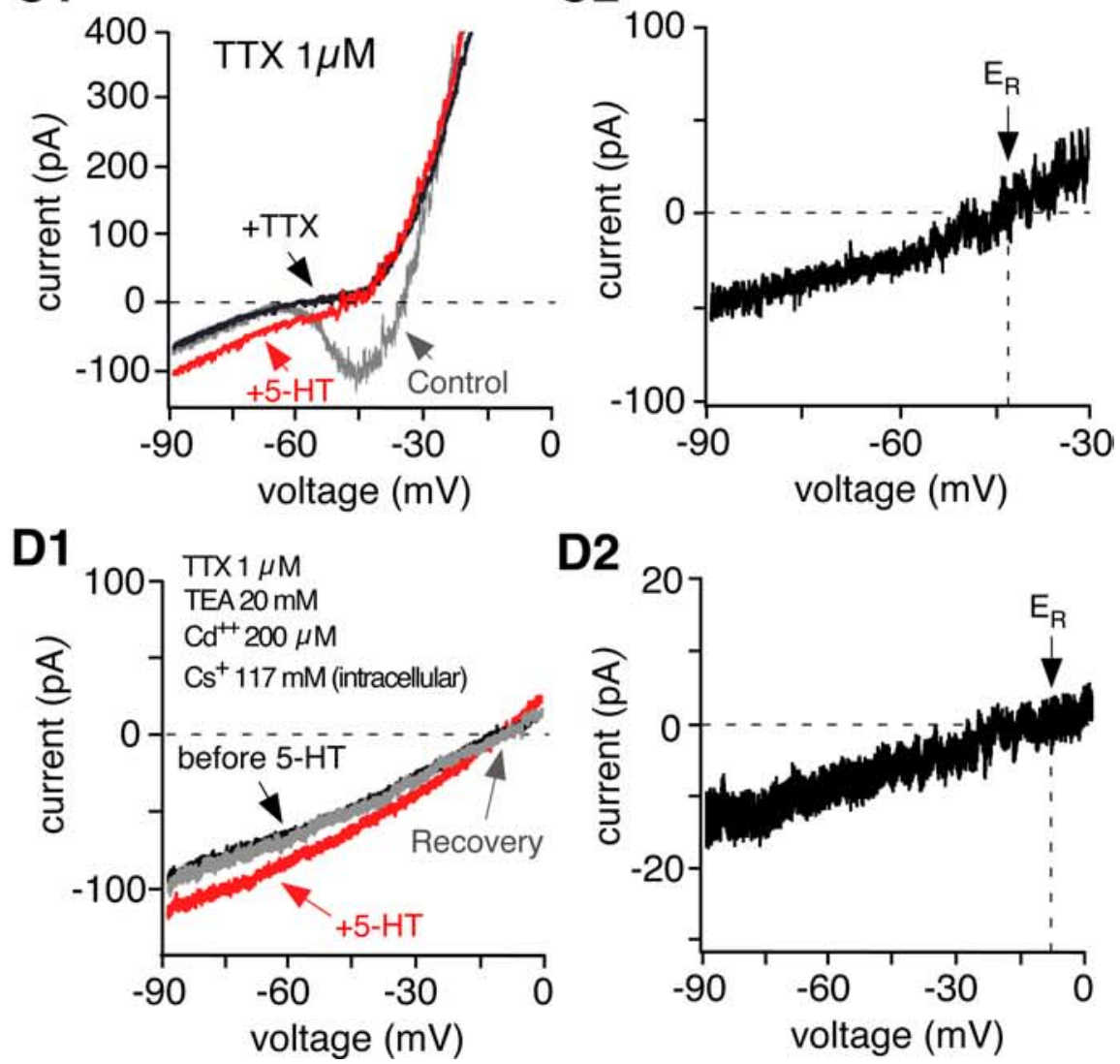

Figure 13. Serotonin depolarizes pre-BötC neurons in part by activating a nonselective cation current. $A$, XII inspiratory activity (top, $\int$ XII) recorded simultaneously with membrane potential of a pre-BötC neuron (bottom). At the network level, bath-applied 5-HT $(30 \mu \mathrm{M})$ increased inspiratory frequency. There was also increased motoneuron tonic activity as seen by the upward shift in baseline of the integrated XII population activity. At the cellular level, 5-HT depolarized the pre-BötC neuron. The depolarization rent observed here (Fig. 13D) and therefore is a candidate for the non- $\mathrm{K}^{+}$component of 5-HT-induced current. Genetic deletion of NALCN is fatal in the neonatal period, due to abnormal breathing ( $\mathrm{Lu}$ et al., 2007). Our novel finding that an NALCN-like current is modulated by 5-HT may explain the dependence of stable breathing on NALCN.

We also found that SP induces a net inward cation leak current (Hayes and Del Negro, 2007) in pre-BötC neurons, although it is not yet clear whether this current(s) is the same as that activated by 5 -HT or the SP-induced $\mathrm{Na}^{+}$-dependent leak current observed in mouse pre-BötC neurons (Peña and Ramirez, 2004). Furthermore, SP also modulates a $\mathrm{K}^{+}$dominated leak current in some pre-BötC neurons (Koizumi and Smith, 2008), indicating heterogeneity.

Serotonin, but not SP, transformed some nonintrinsically bursting pre-BötC inspiratory neurons into intrinsic bursters in vitro. Enhancement of pre-BötC neuron intrinsic bursting by SP in concert with NMDA receptor activation has been reported (Peña et al., 2004), but conversion to intrinsic bursting behavior by SP alone has not (Koizumi and Smith, 2008). Interestingly, it has been shown previously that $\mathrm{TRH}$, which is also contained in and released by 5-HT neurons (Iverfeldt et al., 1989; Richerson, 2004), can induce bursting pacemaker activity in the respiratory portion of the solitary tract nucleus (Dekin et al., 1985), suggesting that 5-HT neurons may enhance respiratory output by inducing bursting behavior of neurons, possibly distributed widely in the respiratory network. We currently do not know whether the actions of 5-HT on leak conductances

induced ectopic bursts (examples indicated by *) out of phase with XII motor output, indicative of an intrinsically bursting neuron. $\boldsymbol{B} 1$, Whole-cell $I-V$ relations recorded in voltage clamp from an intrinsically bursting pre-BötC neuron during slow voltage ramps $(30 \mathrm{mV} / \mathrm{s})$ with (red trace) and without (black trace) bath-applied 5-HT (30 $\mu \mathrm{M}$ ). Rhythmic excitatory synaptic transmission was blocked by CNQX $(20 \mu \mathrm{M})$. Reversal potential for the total leak current $\left(E_{\text {Leak }}\right)$ before 5-HT application is indicated, estimated by extrapolating the linear region of $I-V$ relation after linear regression (dashed line). B2, 5 -HT-induced current obtained by subtracting the $I-V$ curves shown in $\boldsymbol{B}$. The reversal potential $\left(E_{R}\right)$ in this example was near $-35 \mathrm{mV}$. $\mathbf{C 1}, I-V$ relations obtained as in $\boldsymbol{B} 1$ under control conditions (gray), in bath-applied TTX (1 $\mu \mathrm{m}$; black), and in TTX with 5-HT (red). C2,5-HT induced current in TTX ( $E_{\mathrm{R}} \approx$ $-42 \mathrm{mV}) . \mathbf{D 1}, \mathrm{I}-\mathrm{V}$ relations obtained with bath-applied TTX, TEA, and $\mathrm{Cd}^{2+}$ and $\mathrm{Cs}^{+}$-based internal solution. D2, The 5-HT-induced current (difference current) had an $E_{\mathrm{R}} \approx-7.5$ $\mathrm{mV}$, which was identical to total leak reversal potential obtained before 5-HT application. 


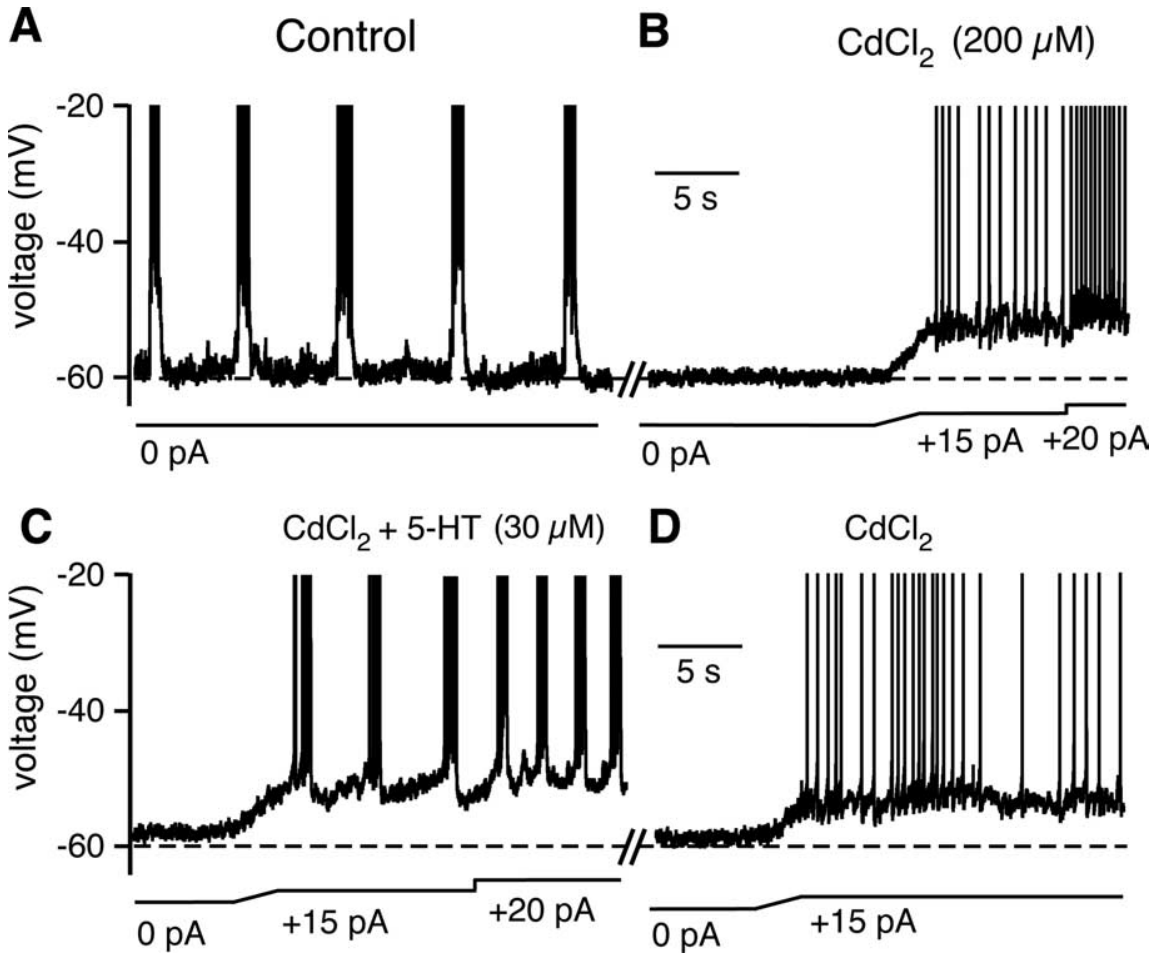

Figure 14. Serotonin converts some nonintrinsically bursting inspiratory pre-BötC neurons into intrinsically bursting cells. $\boldsymbol{A}_{\boldsymbol{t}}$ Current-clamp recording from an inspiratory pre-BötC neuron that burst in phase with inspiratory XII nerve output (data not shown). $\boldsymbol{B}$, After bath-applied $\mathrm{Cd}^{2+}$ to block synaptic transmission, this neuron became silent. In response to applied depolarizing current, the neuron spiked tonically, but did not burst rhythmically. This response is characteristic of pre-BötC nonintrinsically bursting neurons. C, Subsequent bath application of 5- $\mathrm{HT}(30 \mu \mathrm{m})$ transformed the neuron into an intrinsic rhythmic burster. Note the increase in intrinsic bursting frequency at higher applied current, indicating voltage-dependent bursting properties. $\boldsymbol{D}$, Washout of 5-HT caused transformation back to a nonbursting neuron.

alone accounts for the transformation to the intrinsic bursting electrophysiological phenotype, for example by altering leak conductances in relation to persistent sodium conductance in preBötC neurons (Koizumi and Smith, 2008). This conversion may also involve modulation of other conductances, such as voltageactivated $\mathrm{K}^{+}$conductances (Rybak et al., 2003), which could collectively promote intrinsic bursting and rhythmicity of the pre-BötC.

\section{Physiological and pathological significance}

The results presented here from reduced preparations are likely to be relevant to respiratory neural control in vivo. However, there are some differences between our results and those others have reported from in vivo experiments. For example, methysergide and ketanserin do not decrease ventilation when given systemically in vivo in adult animals (Ling et al., 2001; McGuire et al., 2004), and similarly, baseline ventilation is normal in adult mice that lack all 5-HT neurons (Hodges et al., 2008). There are several possible explanations for this discrepancy. Raphé 5-HT neurons are obviously only one component of a set of multiple interacting modulatory control systems that maintain breathing in vivo (Feldman et al., 2003), and other sources of tonic excitatory drive, such as from $\mathrm{CO}_{2} / \mathrm{pH}$-sensitive (chemosensory) glutamatergic neurons (Guyenet et al., 2005), noradrenergic, cholinergic, and orexinergic neurons, may predominate in vivo or compensate for attenuated serotonergic inputs when there is loss of $5-\mathrm{HT}$ throughout development (Hodges et al., 2008). Thus 5- $\mathrm{HT}_{2 \mathrm{~A}}$ receptor antagonists alone may not perturb breathing in vivo simply because effector mechanisms coupled to these receptors, such as the leak channels identified here, could be already predominantly engaged by other modulatory transmitters. Our reduced preparations undoubtedly lack some of the modulatory inputs active in vivo. On the other hand, the sort of detailed pharmacological studies involving systemic administration of progressive doses of 5-HT or NK-1 receptor antagonists that was possible with our mature rodent in situ perfused brainstem-spinal cord preparations have not yet been conducted in mature in vivo preparations. It may be particularly important to determine how combined block of 5-HT and NK-1 receptors affects breathing in vivo since our results indicate that both receptors must be blocked to eliminate respiratory output in the intact brainstem. Such in vivo studies would be beneficial to more fully understand how endogenous activation of 5-HT and NK-1 receptors contribute to stable breathing in vivo, particularly since we also recently found severe disturbances of breathing and increased mortality in neonatal mutant mice lacking raphé 5-HT neurons (M. R. Hodges, M. Wehner, J. Aungst, J. C. Smith, and G. B. Richerson, unpublished observations). Such observations are consistent with our conclusions of a vital role for endogenously released serotonin and SP at least in the neonatal system, and also suggest that further in vivo studies in mature animals will be important for unraveling the developmental contributions of the raphé 5-HT/SP system.

Our results also have implications for disorders in which there is a link between breathing and raphé neurons, such as SIDS, which may involve a defect in the brainstem 5-HT system (Paterson et al., 2006), and sleep apnea, which often involves obstruction of the upper airway due to loss of upper airway muscle tone, including in tongue muscles innervated by XII motoneurons, especially during REM sleep (Kubin et al., 1998) when the firing rate of 5-HT neurons is a minimum (Jacobs and Azmitia, 1992). Withdrawal/alteration of the combination of 5-HT and SP-mediated excitation of the pre-BötC and XII motoneurons has not been considered previously but could contribute to the apneas associated with these conditions.

In summary, the new results presented here provide direct evidence for circuit connections that mediate excitation of respiratory output by the raphé 5-HT/SP system. Our findings indicate that the pre-BötC and raphé obscurus are both part of an interconnected circuit controlling the generation of inspiratory activity. The raphé system augments respiratory circuit activity by functionally concerted mechanisms operating simultaneously at multiple levels of the circuit, including network elements involved in rhythm generation and motor output pattern formation.

\section{References}

Al-Zubaidy ZA, Erickson RL, Greer JJ (1996) Serotonergic and noradrenergic effects on respiratory neural discharge in the medullary slice preparation of neonatal rats. Pflugers Arch 431:942-949. 
Bayliss DA, Umemiya M, Berger AJ (1995) Inhibition of N- and P-type calcium currents and the after-hyperpolarization in rat motoneurones by serotonin. J Physiol 485:635-647.

Bayliss DA, Li YW, Talley EM (1997) Effects of serotonin on caudal raphé neurons: inhibition of $\mathrm{N}$ - and P/Q-type calcium channels and the afterhyperpolarization. J Neurophysiol 77:1362-1374.

Bobker DH, Williams JT (1989) Serotonin augments the cation current $\mathrm{I}_{\mathrm{h}}$ in central neurons. Neuron 2:1535-1540.

Butera RJ Jr, Rinzel J, Smith JC (1999a) Models of respiratory rhythm generation in the pre-Bötzinger complex. I. Bursting pacemaker neurons. J Neurophysiol 82:382-397.

Butera RJ Jr, Rinzel J, Smith JC (1999b) Models of respiratory rhythm generation in the pre-Bötzinger complex. II. Populations of coupled pacemaker neuron. J Neurophysiol 82:398-415.

Carr DB, Cooper DC, Ulrich SL, Spruston N, Surmeier DJ (2002) Serotonin receptor activation inhibits sodium current and dendritic excitability in prefrontal cortex via a protein kinase C-dependent mechanism. J Neurosci 22:6846-6855.

Dean C, Marson L, Kampine JP (1993) Distribution and co-localization of 5-hydroxytryptamine, thyrotropin-releasing hormone and substance $\mathrm{P}$ in the cat medulla. Neuroscience 57:811-822.

Dekin MS, Richerson GB, Getting PA (1985) Thyrotropin-releasing hormone induces rhythmic bursting in neurons of the nucleus tractus solitarius. Science 229:67-69.

Del Negro CA, Johnson SM, Butera RJ, Smith JC (2001) Models of respiratory rhythm generation in the pre-Bötzinger complex. III. Experimental tests of model predictions. J Neurophysiol 86:59-74.

Del Negro CA, Koshiya N, Butera RJ Jr, Smith JC (2002) Persistent sodium current, membrane properties and bursting behavior of pre-Bötzinger complex inspiratory neurons in vitro. J Neurophysiol 88:2242-2250.

Del Negro CA, Morgado-Valle C, Hayes JA, Mackay DD, Pace RW, Crowder EA, Feldman JL (2005) Sodium and calcium current-mediated pacemaker neurons and respiratory rhythm generation. J Neurosci 25:446-453.

Feldman JL, Mitchell GS, Nattie EE (2003) Breathing: rhythmicity, plasticity, chemosensitivity. Annu Rev Neurosci 26:239-266.

Funk GD, Smith JC, Feldman JL (1993) Generation and transmission of respiratory oscillations in medullary slices: role of excitatory amino acids. J Neurophysiol 70:1497-1515.

Gasparini S, DiFrancesco D (1999) Action of serotonin on the hyperpolarization-activated cation current (Ih) in rat CA1 hippocampal neurons. Eur J Neurosci 11:3093-3100.

Gray PA, Janczewski WA, Mellen N, McCrimmon DR, Feldman JL (2001) Normal breathing requires preBötzinger complex neurokinin-1 receptorexpressing neurons. Nat Neurosci 4:927-930.

Guyenet PG, Mulkey DK, Stornetta RL, Bayliss DA (2005) Regulation of ventral surface chemoreceptors by the central respiratory pattern generator. J Neurosci 25:8938-8947.

Hayes JA, Del Negro CA (2007) Neurokinin receptor-expressing preBötzinger complex neurons in neonatal mice studied in vitro. J Neurophysiol 97:4215-4224.

Hodges MR, Tattersall GJ, Harris MB, McEvoy SD, Richerson DN, Deneris ES, Johnson RL, Chen ZF, Richerson GB (2008) Defects in breathing and thermoregulation in mice with near-complete absence of central serotonin neurons. J Neurosci 28:2495-2505.

Hwang LL, Dun NJ (1999) 5-HT modulates multiple conductances in immature rat rostral ventrolateral medulla neurons in vitro. J Physiol 517:217-228.

Iverfeldt K, Serfözö P, Diaz Arnesto L, Bartfai T (1989) Differential release of coexisting neurotransmitters: frequency dependence of the efflux of substance $\mathrm{P}$, thyrotropin releasing hormone and $[3 \mathrm{H}]$ serotonin from tissue slices of rat ventral spinal cord. Acta Physiol Scand 137:63-71.

Jacobs BL, Azmitia EC (1992) Structure and function of the brain serotonin system. Physiol Rev 72:165-229.

Koizumi H, Smith JC (2008) Persistent $\mathrm{Na}^{+}$and $\mathrm{K}^{+}$-dominated leak currents contribute to inspiratory rhythm generation in the pre-BötC in vitro. J Neurosci 27:1773-1785

Koizumi H, Wilson CG, Wong S, Yamanishi T, Koshiya N, Smith JC (2008) Functional imaging, spatial reconstruction and biophysical analysis of a respiratory motor circuit isolated in vitro. J Neurosci 28:2353-2365.

Koshiya N, Smith JC (1999) Neuronal pacemaker for breathing visualized in vitro. Nature 400:360-363.
Kubin L, Davies RO, Pack AI (1998) Control of upper airway motoneurons during REM sleep. News Physiol Sci 13:91-97.

Lalley PM (1986) Serotonergic and non-serotonergic responses of phrenic motoneurons to raphe stimulation in the cat. J Physiol 380:373-385.

Lalley PM, Bischoff AM, Richter DW (1994) Serotonin 1A-receptor activation suppresses respiratory apneusis in the cat. Neurosci Lett 172:59-62.

Lindsey BG, Hernandez YM, Morris KF, Shannon R (1992) Functional connectivity between brain stem midline neurons with respiratorymodulated firing rates. J Neurophysiol 67:890-904.

Ling L, Fuller DD, Bach KB, Kinkead R, Olson EB Jr, Mitchell GS (2001) Chronic intermittent hypoxia elicits serotonin-dependent plasticity in the central neural control of breathing. J Neurosci 21:5381-5388.

Lu B, Su Y, Das S, Liu J, Xia J, Ren D (2007) The neuronal channel NALCN contributes resting sodium permeability and is required for normal respiratory rhythm. Cell 129:371-383.

Manzke T, Guenther U, Ponimaskin EG, Haller M, Dutschmann M, Schwarzacher S, Richter DW (2003) 5- $\mathrm{HT}_{4(\mathrm{a})}$ receptors avert opioid-induced breathing depression without loss of analgesia. Science 301:226-229.

Mason P (1997) Physiological identification of pontomedullary serotonergic neurons in the rat. J Neurophysiol 77:1087-1098.

McGuire M, Zhang Y, White DP, Ling L (2004) Serotonin receptor subtypes required for ventilatory long-term facilitation ad its enhancement after chronic intermittent hypoxia in awake rats. Am J Physiol Regul Integr Comp Physiol 286:R334-R341.

Menetrey D, Basbaum AI (1987) The distribution of substance P-, enkephalin- and dynorphin-immunoreactive neurons in the medulla of the rat and their contribution to bulbospinal pathways. Neuroscience 23:173-187.

Morgado-Valle C, Feldman JL (2004) Depletion of substance P and glutamate by capsaicin blocks respiratory rhythm in neonatal rat in vitro. J Physiol 555:783-792.

Olson EB Jr, Dempsey JA, McCrimmon DR (1979) Serotonin and the control of ventilation in awake rats. J Clin Invest 64:689-693.

Pace RW, Mackay DD, Feldman JL, Del Negro CA (2007) Role of persistent sodium current in mouse preBötzinger complex neurons and respiratory rhythm generation. J Physiol 580:485-496.

Paterson DS, Trachtenberg FL, Thompson EG, Belliveau RA, Beggs AH, Darnall R, Chadwick AE, Krous HF, Kinney HC (2006) Multiple serotonergic brainstem abnormalities in sudden infant death syndrome. JAMA 296:2124-2132.

Paton JFR (1996) A working heart-brainstem preparation of the mouse. J Neurosci Methods 65:63-68.

Peever JH, Necakov A, Duffin J (2001) Nucleus raphé obscurus modulates hypoglossal output of neonatal rat in vitro transverse brain stem slices. J Appl Physiol 90:269-279.

Peña F, Ramirez JM (2002) Endogenous activation of serotonin-2A receptors is required for respiratory rhythm generation in vitro. J Neurosci 22:11055-11064.

Peña F, Ramirez JM (2004) Substance P-mediated modulation of pacemaker properties in the mammalian respiratory network. J Neurosci 24:7549-7556.

Peña F, Parkis MA, Tryba AK, Ramirez JM (2004) Differential contribution of pacemaker properties to the generation of respiratory rhythms during normoxia and hypoxia. Neuron 43:105-117.

Pickering AE, Paton JFR (2006) A decerebrate, artificially-perfused in situ preparation of rat: utility for the study of autonomic and nociceptive processing. J Neurosci Methods 155:260-271.

Purvis LK, Smith JC, Koizumi H, Butera RJ (2007) Intrinsic bursters increase the robustness of rhythm generation in an excitatory network. J Neurophysiol 97:1515-1526.

Richerson GB (2004) Serotonergic neurons as carbon dioxide sensors that maintain pH homeostasis. Nat Rev Neurosci 5:449-461.

Richter DW, Manzke T, Wilken B, Ponimaskin E (2003) Serotonin receptors: guardians of stable breathing. Trends Mol Med 9:542-548.

Rybak IA, Shevtsova NA, St.-John WM, Paton JFR, Pierrefiche O (2003) Endogenous rhythm generation in the pre-Bötzinger complex and ionic currents: modelling and in vitro studies. Eur J Neurosci 18:239-257.

Schwarzacher SW, Pestean A, Günther S, Ballanyi K (2002) Serotonergic modulation of respiratory motoneurons and interneurons in brainstem slices of perinatal rats. Neuroscience 115:1247-1259.

Smith JC, Ellenberger HH, Ballanyi K, Richter DW, Feldman JL (1991) Pre- 
Bötzinger complex: a brainstem region that may generate respiratory rhythm in mammals. Science 254:726-729.

Smith JC, Abdala APL, Koizumi H, Rybak IA, Paton JFR (2007) Spatial and functional architecture of the mammalian brainstem respiratory network: a hierarchy of three oscillatory mechanisms. J Neurophysiol 98:3370-3387.

St.-John WM, Leiter JC (2008) Maintenane of gasping and restoration of eupnea after hypoxia is impaired following blockers of alpha1adrenergic receptors and serotonin 5-HT2 receptors. J Appl Physiol 104:665-673.

Stornetta RL, Rosin DL, Wang H, Sevigny CP, Weston MC, Guyenet PG (2003) A group of glutamatergic interneurons expressing high levels of both neurokinin-1 receptors and somatostatin identifies the region of the pre-Bötzinger complex. J Comp Neurol 455:499-512.

Talley EM, Lei Q, Sirois JE, Bayliss DA (2000) TASK-1, a two-pore domain $\mathrm{K}+$ channel, is modulated by multiple neurotransmitters in motoneurons. Neuron 25:399-410.
Talley EM, Solorzano G, Lei Q, Kim D, Bayliss DA (2001) CNS distribution of members of the two-pore-domain (KCNK) potassium channel family. J Neurosci 21:7491-7505.

Toppin VAL, Harris MB, Kober AM, Leiter JC, St.-John WM (2007) Persistence of eupnea and gasping following blockade of both serotonin type 1 and 2 receptors in the in situ juvenile rat preparation. J Appl Physiol 103:220-227.

Vandermaelen CP, Aghajanian GK (1983) Electrophysiological and pharmacological characterization of serotonergic dorsal raphé neurons re corded extracellularly and intracellularly in rat brain slices. Brain Res 289:109-119.

Wang W, Tiwari JK, Bradley SR, Zaykin RV, Richerson GB (2001) Acidosisstimulated neurons of the medullary raphé are serotonergic. J Neurophysiol 85:2224-2235.

Xiang Z, Wang L, Kitai ST (2005) Modulation of spontaneous firing in rat subthalamic neurons by 5 -HT receptor subtypes. J Neurophysiol 93:1145-1157. 